\title{
SINGULAR LIMITS FOR 4-DIMENSIONAL GENERAL STATIONARY Q-KURAMOTO-SIVASHINSKY EQUATION (Q-KSE) WITH EXPONENTIAL NONLINEARITY
}

\section{TAIEB OUNI, SAMI BARAKET AND MOUFIDA KHTAIFI}

\begin{abstract}
Let $\Omega$ be a bounded domain in $\mathbb{R}^{4}$ with smooth boundary, and let $x^{1}, x^{2}, \cdots, x^{m}$ be points in $\Omega$. We are concerned with the singular stationary non-homogenous q-Kuramoto-Sivashinsky eaquation (q-KSE):$$
\Delta^{2} u-\gamma \Delta u-\lambda|\nabla u|^{q}=\rho^{4} e^{u}
$$

where we use some nonlinear domain decomposition method to give a sufficient condition to have a positive weak solution $u$ in $\Omega$ under the physical Dirichlet-like boundary conditions $u=\Delta u=0$ on $\partial \Omega$, which is singular at each $x^{i}$ as the parameters $\lambda, \gamma$ and $\rho$ tend to 0 and where $q \in[1,4]$ is a real number.
\end{abstract}

Key Words: Singular limits, Green's function, Kuramoto-Sivashinsky equation, Exponential nonlinearity, Nonlinear domain decomposition method.

2010 Mathematics Subject Classification: 58J08, 35J40, 35J60, 35J75.

Received: 03.10.2015

Revised: 05.12 .2015

Accepted: 16.12.2015 
SINGULAR LIMITS FOR 4-DIMENSIONAL GENERAL STATIONARY

Q-KURAMOTO-SIVASHINSKY EQUATION (Q-KSE) WITH EXPONENTIAL

NONLINEARITY

\section{Introduction and statement of the results}

A fundamental goal in the study of non-linear initial boundary value problems involving partial differential equations is to determine whether solutions to a given equation develop a singularity. Resolving the issue of blow-up is important, in part because it can have bearing on the physical relevance and validity of the underlying model. However, determining the answer to this question is notoriously difficult for a wide range of equations such fourth order equation like stationary non homogenous general Kuramoto-Sivashinsky equation with strong nonlinearity like exponential $e^{u}$ :

$$
\Delta^{2} u-\gamma \Delta u-\lambda|\nabla u|^{q}=\rho^{4} e^{u} .
$$

One route is to try to simplify or modify the boundary conditions in an attempt to gain evidence for or against the occurrence of blow-up. A second route is to modify the equations in some way, and to study the modified equations with the hope of gaining insight into the blow-up of solutions to the original equations: see problems (3)-(11) below and the effect of the presence of the second-order backward diffusion term $-\gamma \Delta u$ and the nonlinear term $-\lambda|\nabla u|^{q}$ in (3). The occurrence and type of blow-up depends on the parameters $\lambda, \gamma$ and the domain. Studying this type of equations, we will answer for different basic questions. We concentrate next on the analysis of the main questions raised in the study of blow-up for such equations. This list can be suitably adapted to other singularity formation problems. We will examine several case related to such approaches where basic list includes the questions of, where and how. We propose here an expanded list of three items: (i) Does blow-up occur? (ii) Where? (iii) How? For the first question, the blow-up problem is properly formulated only when a suitable class of solutions is chosen for all solutions in the given class or only for some solutions (which should be identified) or other kinds of generalized solutions can be more natural to a given problem and which equations and problems do exhibit blow-up. The second question is where finite number of points, or regional blow-up, are localized? For the third question, we are only interested on calculate the rate at which solution diverges as $x$ approaches to the set of blow-up point and to calculate the blow-up profiles as limits of solution at the non-blowing points. A major aim of the present work is to provide examples which demonstrate that one must be extremely cautious in generalizing claims about the blow-up of problems studied in idealized settings to claims about the blow-up of the original problem and to the nonlinearity of a problem which can cause the formation of a singularity, where no such singularity is present in the unaltered equation. However, many such studies have tried to search for singularities of the solutions of the equations in the setting of different types of boundary 
SINGULAR LIMITS FOR 4-DIMENSIONAL GENERAL STATIONARY

Q-KURAMOTO-SIVASHINSKY EQUATION (Q-KSE) WITH EXPONENTIAL

NONLINEARITY

conditions like periodic boundary conditions related to the solution of general Kuramoto-Sivashinsky equation. The question of blow up of solutions of stationary general Kuramoto-Sivashinsky equation is still an open question in dimensions fourth and in higher cases.

First, we introduce a model arising in the growth of amorphous surfaces which is described by the following partial differential equation, called in the literature the Kuramoto-Sivashinsky equation:

$$
\partial_{t} u+\Delta^{2} u-\gamma \Delta u-\lambda|\nabla u|^{2}=0 .
$$

on $R^{d}$ with $d \geq 1$, where $\lambda$ and $\gamma$ be real parameters. The KuramotoSivashinsky equation has been independently discovered by Kuramoto and Tsuzuki [13], and by Sivashinsky [24] in the study of a reaction-diffusion system and flame front propagation, respectively, as well as in the study of $2 \mathrm{D}$ Kolmogorov fluid flows [25]. This form of the Kuramoto-Sivashinsky equations is sometimes called the integrated version of the Kuramoto-Sivashinsky equations, arises also in several other models for surface growth. This type of version equation is suggested in [17], [18] (and some reference therein...) as a phenomenological model for the growth of an amorphous surface $\left(Z_{r 65} A l_{7,5}, C u_{27,5}\right)$.

One can also consider a generalization of (1), called in the literature the hyper-viscous Hamilton Jacobian:

$$
\partial_{t} u+\Delta^{2} u-\gamma \Delta u-\lambda|\nabla u|^{q}=0
$$

on the whole $\mathbb{R}^{d}, d \geq 1$, where $\lambda$ and $\gamma$ be real parameters. This equation for $q>2$ and $(\gamma, \lambda)=\left(1, \frac{1}{2}\right)$ was considered in [5], where it was shown that when $q>2$, under the boundary conditions $u=\Delta u=0$ on $\partial \Omega$, a singularity develops in finite-time, provided that the initial data is sufficiently large in a certain sense. (In fact, in [5], the authors proved an even stronger result, as they did not need the destabilizing term $\Delta u$.)

The global regularity for the higher dimensional stationary KuramotoSivashinsky equations is a remarkable open question in nonlinear analysis. Inspired by this question, we introduce in this paper a family of stationary hyper-viscous Hamilton-Jacobi-like equations parameterized by the exponent in the nonlinear term, $q$, called in the literature by the general non homogenous stationary Kuramoto-Sivashinsky equation:

$$
\Delta^{2} u-\gamma \Delta u-\lambda|\nabla u|^{q}=f(u)
$$

in $\Omega \subset \mathbb{R}^{4}$ under the physical Dirichlet-like boundary conditions $u=\Delta u=0$ on $\partial \Omega$ and certain nonlinearity $f(u)$. We refer the reader to [8] where the 
SINGULAR LIMITS FOR 4-DIMENSIONAL GENERAL STATIONARY

Q-KURAMOTO-SIVASHINSKY EQUATION (Q-KSE) WITH EXPONENTIAL

NONLINEARITY

author give some explicit estimates for the $L^{\infty}$-norm of the periodic solutions of the time-independent non-homogeneous Kuramoto-Sivashinsky equation in $\mathbb{R}$ in case $q=1$. In particular, they give an estimate of the Michelson's upper bound of all periodic solutions of the time-independent homogeneous Kuramoto-Sivashinsky equation.

One of the purpose of the present paper is to present a rather efficient method to solve such singularly perturbed problems of the time-independent general Kuramoto-Sivashinsky equation. This method has already been used successfully in geometric context(constant mean curvature surfaces, constant scalar curvature metrics, extremal Kähler metrics, manifolds with special holonomy, ...) the first time has appeared in the context of partial differential equations is given in [1]. We felt that, given the interest in singular perturbation problems, it is worth illustrating this on the non-Homogenous stationary q-Kuramoto-Sivashinsky equation: $\Delta^{2} u-\gamma \Delta u-\lambda|\nabla u|^{q}=f(u)$ in $\Omega \subset \mathbb{R}^{4}$ under the physical Dirichlet-like boundary conditions $u=\Delta u=0$ on $\partial \Omega$ and certain nonlinearity $f(u)$ given by the following problem :

Let $\Omega \subset \mathbb{R}^{4}$ be a regular bounded open domain in $\mathbb{R}^{4}$. We are interested in positive solutions of the general stationary q-Kuramoto-Sivashinsky problem:

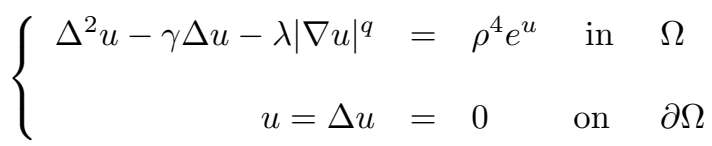

when the parameters $\rho, \lambda$ and $\gamma$ tend to 0 and $q \in[1,4]$. Problem $(3)_{\mid q=2}$ has been treated by first author et al in [4] in context of blow up. For $\lambda=0$, we refer the reader to [9], where the author consider the problem, without non linear gradient term:

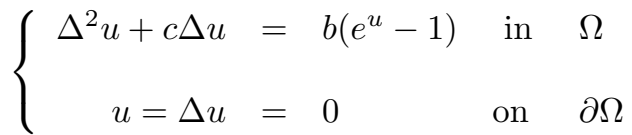

where $\Omega$ is a bounded and smooth domain of $\mathbb{R}^{n}, c \in \mathbb{R}$ and $b \in \mathbb{R}$. The author prove some existence and nonexistence results for (4) via variational techniques. Such equations may occur while studying traveling waves in suspension bridges. For more general problem see [21], for the following Navier boundary value problem:

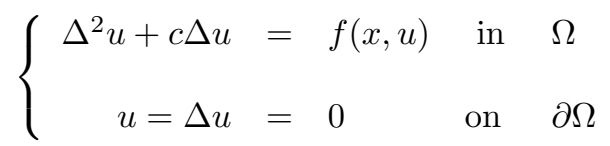


SINGULAR LIMITS FOR 4-DIMENSIONAL GENERAL STATIONARY

in $\mathbb{R}^{n}, n \geq 4$ and $f$ is non linear growth function. Using the Sobolev compact embedding $H^{2}(\Omega) \cap H_{0}^{1}(\Omega) \hookrightarrow L^{q}(\Omega)\left(1 \leq q<p^{*}\right.$, where $\left.p^{*}=\frac{2 n}{n-4}\right)$, it is easy to see that seeking a weak solution of problem (5) is equivalent to finding a nonzero critical points of the following functional on $E:=H^{2}(\Omega) \cap H_{0}^{1}(\Omega)$ :

$$
I:=\frac{1}{2} \int_{\Omega}\left(|\Delta u|^{2}-c|\nabla u|^{2}\right) d x-\int_{\Omega} F(x, u) \text { where } F(x, u)=\int_{0}^{u} F(x, t) d t .
$$

In conformal dimensional a.e $n=4$ and $f$ has the subcritical (exponential) growth on $\Omega$, a.e.,

$$
\lim _{t \rightarrow+\infty} \frac{|f(x, t)|}{\exp (\alpha t)}=0
$$

uniformly on $x \in \Omega$ for all $\alpha>0$ and in some cases and hypothesis and using Adams inequality, (see [20]), for the fourth-order derivative, namely,

$$
\sup _{\{u \in E,\|u\| \leq 1\}} \int_{\Omega} e^{32 \pi^{2} u^{2}} d x \leq C|\Omega|,
$$

where $\|u\|=: \int_{\Omega}\left(|\Delta u|^{2}-c|\nabla u|^{2}\right)^{1 / 2} d x$, the authors show that the problem (5) has at least two nontrivial solutions (for more details see Theorem 1.3 in [21]) or infinitely many nontrivial solutions (for more details see Theorem 1.4 in [21]).

Problem (3) can be considered as a higher order counterpart of the

$$
\left\{\begin{array}{rlll}
-\Delta u-\lambda|\nabla u|^{q} & =\rho^{2} e^{u} & \text { in } \quad \Omega \subset \mathbb{R}^{2} \\
u & =0 & \text { on } \quad \partial \Omega
\end{array}\right.
$$

when the parameters $\rho$ tends to $0 . \rho^{2}=\frac{8 \varepsilon^{2}}{\left(\varepsilon^{2}+1\right)^{2}} \sim \varepsilon^{2}$ as $\varepsilon$ tends to 0 . This last problem with $q \in[1,2]$, poses as well a number of fundamental mathematical questions in singularity problem (see for example [3]) where the authors do not consider any condition like (A) (see below), to solve such singularity problem. This type of equation is the stationary case of generalized non homogenous viscous Hamilton-Jacobian equations [22]

$$
\left\{\begin{array}{rlll}
\partial_{t} u-\Delta u-\lambda|\nabla u|^{q} & =f(u) & \text { in } \quad & \Omega \\
u & =0 & \text { on } & \partial \Omega
\end{array}\right.
$$

where $\Omega$ is a smooth bounded domain in $\mathbb{R}^{q}, q \geq 1$.

As observed by Ren and Wei in [19], the problem $(6)_{\mid q=2}$, can be reduced to a problem without gradient term. Indeed, if $u$ is a solution of $(6)_{\mid q=2}$, then the function

$$
w=\left(\lambda \rho^{2} e^{u}\right)^{\lambda}
$$


SINGULAR LIMITS FOR 4-DIMENSIONAL GENERAL STATIONARY

Q-KURAMOTO-SIVASHINSKY EQUATION (Q-KSE) WITH EXPONENTIAL

satisfies

$$
\left\{\begin{aligned}
-\Delta w & =w^{\frac{\lambda+1}{\lambda}} \text { in } \Omega \\
w & =\left(\lambda \rho^{2}\right)^{\lambda} \text { on } \partial \Omega,
\end{aligned}\right.
$$

since the exponent $p=\frac{\lambda+1}{\lambda}$ tends to infinity as $\lambda$ tends to 0 , see also [11].

The problem of existence of $v_{\varepsilon, \lambda}$ a sequence of solutions of $(6)_{\mid q=2}$ which converges to some singular function as the parameters $\varepsilon$ and $\lambda$ tend to 0 has studied by Baraket et al. in [2], under the assumption

(A): $\quad$ If $0<\varepsilon<\lambda$, then $\lambda^{1+\delta / 2} \varepsilon^{-\delta} \longrightarrow 0$ as $\lambda \longrightarrow 0$, for any $\delta \in(0,1)$.

( $\rho \sim \varepsilon$ as $\varepsilon$ tends to 0 ). In particular, if we take $\lambda=\mathcal{O}\left(\varepsilon^{2 / 3}\right)$, then condition $(A)$ is satisfied. With assumption $(A)$, problem $(6)_{\mid q=2}$, can be treated as a perturbation of the Liouville equation

$$
-\Delta u=\rho^{2} e^{u} \quad \text { in } \quad \Omega \subset \mathbb{R}^{2} .
$$

The question we would like to study is concerned with the existence of other branches of solutions of (3) which is singular at each $x^{i}, i=1, \cdots, m$ as the parameters $\lambda, \gamma$ and $\rho$ tend to 0 .

To describe our result, let us denote by $G(x, \cdot)$ the solution of

$$
\left\{\begin{aligned}
\Delta^{2} G(x, \cdot) & =64 \pi^{2} \delta_{x} \quad \text { in } \Omega \\
G(x, \cdot) & =\Delta G(x, \cdot)=0 \text { on } \partial \Omega .
\end{aligned}\right.
$$

It is easy to check that the function

$$
R(x, y):=G(x, y)+8 \log |x-y|
$$

is a smooth function.

We define the set of blow-up as

$$
S:=\left\{x \in \Omega: \exists \quad x_{n} \longrightarrow x \quad \text { s.t } \quad u_{n}\left(x_{n}\right) \longrightarrow+\infty\right\}
$$

and

$$
W\left(x^{1}, \ldots, x^{m}\right):=\sum_{j=1}^{m} R\left(x^{j}, x^{j}\right)+\sum_{j \neq \ell} G\left(x^{j}, x^{\ell}\right) .
$$

for $x^{1}, x^{2}, \cdots, x^{m}$ m-points in $\Omega$.

Many papers have been devoted to the case $(\gamma, \lambda)=(0,0)$, where the problem (3) becomes

$$
\left\{\begin{array}{rll}
\Delta^{2} u & =\rho^{4} e^{u} \quad \text { in } \quad \Omega \\
u & =\Delta u=0 \text { on } \partial \Omega
\end{array}\right.
$$


SINGULAR LIMITS FOR 4-DIMENSIONAL GENERAL STATIONARY

when the parameter $\rho$ tends to 0. ( See for example [1]). Semilinear equations involving fourth order elliptic operator and exponential nonlinearity appear naturally in conformal geometry and in particular in the prescription of the so called $Q$-curvature on 4-dimensional Riemannian manifolds [6], [7]

$$
Q_{g}=\frac{1}{12}\left(-\Delta_{g} S_{g}+S_{g}^{2}-3\left|\mathrm{Ric}_{g}\right|^{2}\right)
$$

where Ric $_{g}$ denotes the Ricci tensor and $S_{g}$ is the scalar curvature of the metric $g$. Recall that the $Q$-curvature changes under a conformal change of metric

$$
g_{w}=e^{2 w} g
$$

according to

$$
P_{g} w+2 Q_{g}=2 \tilde{Q}_{g_{w}} e^{4 w}
$$

where

$$
P_{g}:=\Delta_{g}^{2}+\delta\left(\frac{2}{3} S_{g} I-2 \mathrm{Ric}_{g}\right) d
$$

is the Panietz operator, which is an elliptic 4-th order partial differential operator [7] and which transforms according to

$$
e^{4 w} P_{e^{2 w} g}=P_{g}
$$

under a conformal change of metric $g_{w}:=e^{2 w} g$. In the special case where the manifold is the Euclidean space, the Panietz operator is simply given by

$$
P_{g_{\text {eucl }}}=\Delta^{2}
$$

in which case (12) reduces to

$$
\Delta^{2} w=\tilde{Q} e^{4 w}
$$

the solutions of which give rise to conformal metric $g_{w}=e^{2 w} g_{\text {eucl }}$ whose $Q$ curvature is given by $\tilde{Q}$. There is by now an extensive literature about this problem and we refer to [7] and [10] for references and recent developments. In dimension 4, Wei in [26], have studied the behavior of solutions to the following nonlinear eigenvalue problem for the biharmonic operator $\Delta^{2}$ in $\mathbb{R}^{4}$. More precisely, consider the following problem

$$
\left\{\begin{aligned}
\Delta^{2} u & =\lambda f(u) & \text { in } & & \Omega \\
u=\Delta u & =0 & \text { on } & & \partial \Omega
\end{aligned}\right.
$$


SINGULAR LIMITS FOR 4-DIMENSIONAL GENERAL STATIONARY

and $u^{*}$ the solution of

$$
\left\{\begin{aligned}
\Delta^{2} u^{*} & =64 \pi^{2} \sum_{i=1}^{m} \delta_{x^{i}} & & \text { in } \Omega \\
u^{*}=\Delta u^{*} & =0 & & \text { on } \partial \Omega .
\end{aligned}\right.
$$

The author proved the following result

Theorem 1.1. [26] Let $\Omega$ be a smooth bounded domain in $\mathbb{R}^{4}$ and $f$ a smooth nonnegative increasing function such that

$$
e^{-u} f(u) \text { and } e^{-u} \int_{0}^{u} f(s) d s \text { tend to } 1, \text { as } u \longrightarrow+\infty \text {. }
$$

For $u_{\lambda}$ solution of (15), denote by $\Sigma_{\lambda}=\lambda \int_{\Omega} f\left(u_{\lambda}\right) d x$. Then many cases occur:

i) $\Sigma_{\lambda} \longrightarrow 0$ therefore, $\left\|u_{\lambda}\right\|_{L^{\infty}(\Omega)} \longrightarrow 0$ as $\lambda \longrightarrow 0$.

ii) $\Sigma_{\lambda} \longrightarrow+\infty$ then $u_{\lambda} \longrightarrow+\infty$ as $\lambda \longrightarrow 0$.

iii) $\Sigma_{\lambda} \longrightarrow 64 \pi^{2} m$, for some positive integer $m$. Then the limiting function $u^{*}=\lim _{\lambda \longrightarrow 0} u_{\lambda}$ has $m$ blow-up points, $\left\{x^{1}, \ldots, x^{m}\right\}$, where $u_{\lambda}\left(x^{i}\right) \longrightarrow$ $+\infty$ as $\lambda \longrightarrow 0$. Moreover, $\left(x^{1}, \ldots, x^{m}\right)$ is a critical point of $W$.

In the following, we denote by $\varepsilon$ the smallest positive parameter satisfying

$$
\rho^{4}=\frac{384 \varepsilon^{4}}{\left(1+\varepsilon^{2}\right)^{4}}
$$

Remark that $\rho \sim \varepsilon$ as $\varepsilon \longrightarrow 0$.

Denote by $\sigma_{\lambda, \gamma}=\max (\lambda, \gamma)$ and assume that $\varepsilon, \lambda$ and $\gamma$ satisfy

$\left(A_{\varepsilon, \lambda, \gamma}\right): \quad$ If $0<\varepsilon<\sigma_{\lambda, \gamma}$, then $\sigma_{\lambda, \gamma}^{1+\delta / 2} \varepsilon^{-\delta} \longrightarrow 0$ as $\sigma_{\lambda, \gamma} \longrightarrow 0$, for any $\delta \in(0,1)$.

Our main results reads :

Theorem 1.2. Let $q \in[1,4)$ and $\alpha \in(0,1)$. Let $\Omega$ be an open smooth bounded domain in $\mathbb{R}^{4}$ and $S=\left\{x^{1}, \ldots, x^{m}\right\} \subset \Omega$ be a non empty set. Assume that $\left(x^{1}, \ldots, x^{m}\right)$ is a nondegenerate critical point of $W$, then there exist $\rho_{0}>$ $0, \lambda_{0}>0, \gamma_{0}>0$ and $\left\{u_{\rho, \lambda, \gamma}\right\}_{\substack{0<\rho<\rho_{0} \\ 0<\lambda<\lambda_{0} \\ 0<\gamma<\gamma_{0}}}$ a family of solutions of (3), such that

$$
\lim _{\substack{\rho \rightarrow 0 \\ \lambda \rightarrow 0 \\ \gamma \rightarrow 0}} u_{\rho, \lambda, \gamma}=\sum_{j=1}^{m} G\left(x^{j}, \cdot\right)
$$

in $\mathcal{C}_{\text {loc }}^{4, \alpha}\left(\Omega-\left\{x^{1}, \ldots, x^{m}\right\}\right)$. 
SINGULAR LIMITS FOR 4-DIMENSIONAL GENERAL STATIONARY

Theorem 1.3. Let $q=4$ and $\alpha \in(0,1)$. Let $\Omega$ be an open smooth bounded domain in $\mathbb{R}^{4}$ and $S=\left\{x^{1}, \ldots, x^{m}\right\} \subset \Omega$ be a non empty set. Assume that $\varepsilon, \lambda$ and $\gamma$ satisfy the condition $\left(A_{\varepsilon, \lambda, \gamma}\right)$ and $\left(x^{1}, \ldots, x^{m}\right)$ is a nondegenerate critical point of $W$, then there exist $\rho_{0}>0, \lambda_{0}>0, \gamma_{0}>0$ and $\left\{u_{\rho, \lambda, \gamma}\right\}_{\substack{0<\rho<\rho_{0} \\ 0<\lambda<\lambda_{0} \\ 0<\gamma<\gamma_{0}}}$ a family of solutions of (3), such that

$$
\lim _{\substack{\rho \rightarrow 0 \\ \lambda \rightarrow 0 \\ \gamma \rightarrow 0}} u_{\rho, \lambda, \gamma}=\sum_{j=1}^{m} G\left(x^{j}, \cdot\right)
$$

in $\mathcal{C}_{\text {loc }}^{4, \alpha}\left(\Omega-\left\{x^{1}, \ldots, x^{m}\right\}\right)$.

Our result reduces the study of nontrivial branches of solutions of (3) to the search for critical points of the function $W$ defined in (10). Observe that the assumption of the nondegeneracy of the critical point is a rather mild assumption since it is certainly fulfilled for generic choice of the open domain $\Omega$.

We briefly describe the plan of the paper : In Section 2 we discuss rotationally symmetric solutions of (3). In Section 3 we study the linearized operator about the radially symmetric solution defined in the previous section. In Section 4, we recall some Known results about the analysis of the bi-Laplace operator in weighted spaces. Both section strongly use the b-operator which has been developed by Melrose [15] in the context of weighted Sobolev spaces and by Mazzeo [14] in the context of weighted Hölder spaces (see also [16]).

A first nonlinear problem is studied in Section 5 where the existence of an infinite dimensional family of solutions of (3) which are defined on a large ball and which are close to the rotationally symmetric solution is proven. In Section 6 , we prove the existence of an infinite dimensional family of solutions of (3) which are defined on $\Omega$ with small ball removed. Finally, in Section 7 , we show how elements of these infinite dimensional families can be connected to produce solutions of (3) described in Theorem 1.2 and Theorem 1.3. This last section borrows ideas from applied mathematics were domain decomposition methods are of common use. Throughout the paper, the symbol $c_{\kappa}>0$ (which can depend only on $\kappa$ ) denotes always a positive constant independent of $\varepsilon, \lambda$ and $\gamma$ which might change from one line to another.

\section{Rotationally symmetric solutions}

We first describe the rotationally symmetric approximate solutions of

$$
\Delta^{2} u-\gamma \Delta u-\lambda|\nabla u|^{q}=\rho^{4} e^{u}
$$


SINGULAR LIMITS FOR 4-DIMENSIONAL GENERAL STATIONARY

Q-KURAMOTO-SIVASHINSKY EQUATION (Q-KSE) WITH EXPONENTIAL

NONLINEARITY

in $\mathbb{R}^{4}$ for $q \in[1,4]$, which will play a central role in our analysis. For this reason given $\varepsilon>0$, we define

$$
u_{\varepsilon}(x):=4 \log \left(1+\varepsilon^{2}\right)-4 \log \left(\varepsilon^{2}+|x|^{2}\right) .
$$

which is clearly a solution of

$$
\Delta^{2} u-\rho^{4} e^{u}=0
$$

when

$$
\rho^{4}=\frac{384 \varepsilon^{4}}{\left(1+\varepsilon^{2}\right)^{4}} .
$$

Let us notice that equation (19) is invariant under some dilation in the following sense : If $u$ is a solution of (19) and if $\tau>0$, then $u(\tau \cdot)+4 \log \tau$ is also a solution of (19). With this observation in mind, we define, for all $\tau>0$

$$
u_{\varepsilon, \tau}(x):=4 \log \left(1+\varepsilon^{2}\right)+4 \log \tau-4 \log \left(\varepsilon^{2}+\tau^{2}|x|^{2}\right) .
$$

\section{A linear fourth order elliptic operator on $\mathbb{R}^{4}$}

We define the linear fourth order elliptic operator

$$
\mathbb{L}:=\Delta^{2}-\frac{384}{\left(1+|x|^{2}\right)^{4}}
$$

which corresponds to the linearization of (19) about the solution $u_{1}\left(=u_{\varepsilon=1}\right)$ which has been defined in the previous section.

We are interested in the classification of bounded solutions of $\mathbb{L} w=0$ in $\mathbb{R}^{4}$. Some solutions are easy to find. For example, we can define

$$
\phi_{0}(x):=r \partial_{r} u_{1}(x)+4=4 \frac{1-r^{2}}{1+r^{2}},
$$

where $r=|x|$. Clearly $\mathbb{L} \phi_{0}=0$ and this reflects the fact that (19) is invariant under the group of dilations $\tau \longrightarrow u(\tau \cdot)+4 \log \tau$. We also define, for $i=$ $1, \ldots, 4$

$$
\phi_{i}(x):=-\partial_{x_{i}} u_{1}(x)=\frac{8 x_{i}}{1+|x|^{2}},
$$

which are also solutions of $\mathbb{L} \phi_{j}=0$ since these solutions correspond to the invariance of the equation under the group of translations $a \longrightarrow u(\cdot+a)$.

The following result classifies all bounded solutions of $\mathbb{L} w=0$ which are defined in $\mathbb{R}^{4}$. 
SINGULAR LIMITS FOR 4-DIMENSIONAL GENERAL STATIONARY

Lemma 3.1. [1] Any bounded solution of $\mathbb{L} w=0$ defined in $\mathbb{R}^{4}$ is a linear combination of $\phi_{i}$ for $i=0,1, \ldots, 4$.

Let $B_{r}$ denote the ball of radius $r$ centered at the origin in $\mathbb{R}^{4}$.

Definition 3.1. Given $k \in \mathbb{N}, \alpha \in(0,1)$ and $\mu \in \mathbb{R}$, we introduce the Hölder weighted spaces $\mathcal{C}_{\mu}^{k, \alpha}\left(\mathbb{R}^{4}\right)$ as the space of functions $w \in \mathcal{C}_{\text {loc }}^{k, \alpha}\left(\mathbb{R}^{4}\right)$ for which the following norm

$$
\|w\|_{\mathcal{C}_{\mu}^{k, \alpha}\left(\mathbb{R}^{4}\right)}:=\|w\|_{\mathfrak{C}^{k, \alpha}\left(\bar{B}_{1}\right)}+\sup _{r \geq 1}\left(\left(1+r^{2}\right)^{-\mu / 2}\|w(r \cdot)\|_{\mathfrak{C}^{k, \alpha}\left(\bar{B}_{1}-B_{1 / 2}\right)}\right),
$$

is finite.

More details about these spaces and their use in nonlinear problems can be found in [16]. Roughly speaking, functions in $\mathcal{C}_{\mu}^{k, \alpha}\left(\mathbb{R}^{4}\right)$ are bounded by a constant times $\left(1+r^{2}\right)^{\mu / 2}$ and have their $\ell-t h$ partial derivatives that are bounded by $\left(1+r^{2}\right)^{\mu / 2}$, for $\ell=1, \cdots, k+\alpha$. We also define

$$
\mathcal{C}_{r a d, \mu}^{k, \alpha}\left(\mathbb{R}^{4}\right)=\left\{f \in \mathcal{C}_{\mu}^{k, \alpha}\left(\mathbb{R}^{4}\right) ; f(x)=f(|x|), \forall x \in \mathbb{R}^{4}\right\} .
$$

As a consequence of the result of Lemma 3.1, we have the :

Proposition 3.1. [1] i) Assume that $\mu>1$ and $\mu \notin \mathbb{N}$, then

$$
\begin{aligned}
L_{\mu}: \mathcal{C}_{\mu}^{4, \alpha}\left(\mathbb{R}^{4}\right) & \longrightarrow \mathcal{C}_{\mu-4}^{0, \alpha}\left(\mathbb{R}^{4}\right) \\
w & \longmapsto \mathbb{L} w
\end{aligned}
$$

is surjective.

ii) Assume that $\delta>0$ and $\delta \notin \mathbb{N}$ then

$$
\begin{aligned}
& L_{\text {rad, }, \delta}: \quad \mathcal{C}_{r a d, \delta}^{4, \alpha}\left(\mathbb{R}^{4}\right) \quad \longrightarrow \quad \mathcal{C}_{r a d, \delta-4}^{0, \alpha}\left(\mathbb{R}^{4}\right) \\
& w \quad \longmapsto \quad \mathbb{L} w
\end{aligned}
$$

is surjective.

We set $\bar{B}_{1}^{*}=\bar{B}_{1}-\{0\}$.

Definition 3.2. Given $k \in \mathbb{N}, \alpha \in(0,1)$ and $\mu \in \mathbb{R}$, we introduce the Hölder weighted space $\mathrm{C}_{\mu}^{k, \alpha}\left(\bar{B}_{1}^{*}\right)$ as the space of functions in $\mathrm{C}_{\text {loc }}^{k, \alpha}\left(\bar{B}_{1}^{*}\right)$ for which the following norm

$$
\|u\|_{\mathcal{C}_{\mu}^{k, \alpha}\left(\bar{B}_{1}^{*}\right)}=\sup _{r \leq 1 / 2}\left(r^{-\mu}\|u(r \cdot)\|_{\mathcal{C}^{k, \alpha}\left(\bar{B}_{2}-B_{1}\right)}\right)
$$

is finite. 
SINGULAR LIMITS FOR 4-DIMENSIONAL GENERAL STATIONARY

Q-KURAMOTO-SIVASHINSKY EQUATION (Q-KSE) WITH EXPONENTIAL

NONLINEARITY

Then we define the subspace of radial functions in $\mathrm{C}_{\text {rad, } \delta}^{k, \alpha}\left(\bar{B}_{1}^{*}\right)$ by

$$
\mathcal{C}_{\text {rad, }, \delta}^{k, \alpha}\left(\bar{B}_{1}^{*}\right)=\left\{f \in \mathcal{C}_{\delta}^{k, \alpha}\left(\mathbb{R}^{4}\right) ; f(x)=f(|x|), \forall x \in \bar{B}_{1}^{*}\right\} .
$$

For all $\varepsilon, \tau, \lambda>0$, we define

$$
R_{\varepsilon, \lambda, \gamma}:=\tau r_{\varepsilon, \lambda, \gamma} / \varepsilon
$$

where

$$
r_{\varepsilon, \lambda, \gamma}:=\max (\sqrt{\varepsilon}, \sqrt{\lambda}, \sqrt{\gamma})
$$

We would like to find a solution $u$ of

$$
\Delta^{2} u-\gamma \Delta u-\lambda|\nabla u|^{q}-\rho^{4} e^{u}=0
$$

in $\bar{B}_{r_{\varepsilon, \lambda, \gamma}}$. Using the transformation

$$
v(x)=u\left(\frac{\varepsilon}{\tau} x\right)+8 \log \varepsilon-4 \log \left(\tau\left(1+\varepsilon^{2}\right) / 2\right),
$$

then equation (24) is equivalent to

$$
\Delta^{2} v-\gamma\left(\frac{\varepsilon}{\tau}\right)^{2} \Delta v-\lambda\left(\frac{\varepsilon}{\tau}\right)^{4-q}|\nabla v|^{q}-24 e^{v}=0
$$

in $\bar{B}_{R_{\varepsilon, \lambda, \gamma}}$. Now we look for a solution of (25) of the form

$$
v(x)=u_{1}(x)+h(x)
$$

this amounts to solve

$$
\mathbb{L} h=\frac{384}{\left(1+|x|^{2}\right)^{4}}\left(e^{h}-h-1\right)+\gamma\left(\frac{\varepsilon}{\tau}\right)^{2} \Delta\left(u_{1}+h\right)+\lambda\left(\frac{\varepsilon}{\tau}\right)^{4-q}\left|\nabla\left(u_{1}+h\right)\right|^{q}
$$

in $\bar{B}_{R_{\varepsilon, \lambda, \gamma}}$.

We will need the following definition.

Definition 3.3. Given $\bar{r} \geq 1, k \in \mathbb{N}, \alpha \in(0,1)$ and $\mu \in \mathbb{R}$, the weighted space $\mathrm{C}_{\mu}^{k, \alpha}\left(B_{\bar{r}}\right)$ is defined to be the space of functions $w \in \mathrm{C}^{k, \alpha}\left(B_{\bar{r}}\right)$ endowed with the norm

$$
\|w\|_{\mathcal{C}_{\mu}^{k, \alpha}\left(\bar{B}_{\bar{r}}\right)}:=\|w\|_{\mathfrak{e}^{k, \alpha}\left(B_{1}\right)}+\sup _{1 \leq r \leq \bar{r}}\left(r^{-\mu}\|w(r \cdot)\|_{\mathfrak{C}^{k, \alpha}\left(\bar{B}_{1}-B_{1 / 2}\right)}\right) .
$$


For all $\sigma \geq 1$, we denote by

$$
\mathcal{E}_{\sigma}: \mathcal{C}_{\mu}^{0, \alpha}\left(\bar{B}_{\sigma}\right) \longrightarrow \mathcal{C}_{\mu}^{0, \alpha}\left(\mathbb{R}^{4}\right)
$$

the extension operator defined by

$$
\mathcal{E}_{\sigma}(f)(x)=\left\{\begin{array}{ccc}
f(x) & \text { for } & |x| \leq \sigma \\
\chi\left(\frac{|x|}{\sigma}\right) f\left(\sigma \frac{x}{|x|}\right) & \text { for } & |x| \geq \sigma,
\end{array}\right.
$$

where $t \longmapsto \chi(t)$ is a smooth nonnegative cutoff function identically equal to 1 for $t \leq 1$ and identically equal to 0 for $t \geq 2$. It is easy to check that there exists a constant $c=c(\mu)>0$, independent of $\sigma \geq 1$, such that

$$
\left\|\mathcal{E}_{\sigma}(w)\right\|_{\mathcal{C}_{\mu}^{0, \alpha}\left(\mathbb{R}^{4}\right)} \leq c\|w\|_{\mathcal{C}_{\mu}^{0, \alpha}\left(\bar{B}_{\sigma}\right)} .
$$

We fix

$$
\delta \in(0, \min (1,4-q)) \quad \text { for } q \in[1,4)
$$

and

$$
\delta \in(0,1) \quad \text { for } q=4 .
$$

Denote by $\mathcal{G}_{\delta}$ to be a right inverse of $\mathbb{L}_{r a d, \delta}$ provided by Proposition 3.1. To find a solution of (26) it is enough to find a fixed point $h$, in a small ball of $\mathcal{C}_{\text {rad, }, \delta}^{4, \alpha}\left(\mathbb{R}^{4}\right)$, solution of

$$
h=\aleph(h)
$$

where

$$
\aleph(h):=\mathcal{G}_{\delta} \circ \mathcal{E}_{\delta} \circ \mathfrak{R}(h)
$$

with

$\mathfrak{R}(h)=\frac{384}{\left(1+|x|^{2}\right)^{4}}\left(e^{h}-h-1\right)+\gamma\left(\frac{\varepsilon}{\tau}\right)^{2} \Delta\left(u_{1}+h\right)+\lambda\left(\frac{\varepsilon}{\tau}\right)^{4-q}\left|\nabla\left(u_{1}+h\right)\right|^{q}$.

For $|x|=r$, we have

$$
\begin{aligned}
\mathfrak{R}(0) & =\gamma\left(\frac{\varepsilon}{\tau}\right)^{2} \Delta u_{1}+\lambda\left(\frac{\varepsilon}{\tau}\right)^{4-q}\left|\nabla u_{1}\right|^{q} \\
& =-16 \gamma\left(\frac{\varepsilon}{\tau}\right)^{2} \frac{2+r^{2}}{\left(1+r^{2}\right)^{2}}+8^{q} \lambda\left(\frac{\varepsilon}{\tau}\right)^{4-q} \frac{r^{q}}{\left(1+r^{2}\right)^{q}} .
\end{aligned}
$$

* For $q \in[1,4)$. 
SINGULAR LIMITS FOR 4-DIMENSIONAL GENERAL STATIONARY

Given $\kappa>0$, there exist $c_{\kappa}>0$ (which can depend only on $\kappa$ ), such that for $\delta \in(0, \min (1,4-q))$ and $q \in[1,4)$, we have

$$
\begin{aligned}
\sup _{r \leq R_{\varepsilon, \lambda, \gamma}} r^{4-\delta} \mid & \left.\Re(0)\left|\quad \leq c_{\kappa} \gamma\left(\frac{\varepsilon}{\tau}\right)^{2} \sup _{r \leq R_{\varepsilon, \lambda, \gamma}} r^{4-\delta} \Delta u_{1}+c_{\kappa} \lambda\left(\frac{\varepsilon}{\tau}\right)^{4-q} \sup _{r \leq R_{\varepsilon, \lambda, \gamma}} r^{4-\delta}\right| \nabla u_{1}\right|^{q} \\
& \leq c_{\kappa} \gamma\left(\frac{\varepsilon}{\tau}\right)^{2} \sup _{r \leq R_{\varepsilon, \lambda, \gamma}} r^{4-\delta} \frac{2+r^{2}}{\left(1+r^{2}\right)^{2}}+c_{\kappa} \lambda\left(\frac{\varepsilon}{\tau}\right)^{4-q} \sup _{r \leq R_{\varepsilon, \lambda, \gamma}} r^{4-\delta} \frac{r^{q}}{\left(1+r^{2}\right)^{q}} \\
& \leq c_{\kappa} \gamma\left(\frac{\varepsilon}{\tau}\right)^{2} \sup _{r \leq R_{\varepsilon, \lambda, \gamma}} r^{4-\delta} \frac{2}{\left(1+r^{2}\right)^{2}}+c_{\kappa} \gamma\left(\frac{\varepsilon}{\tau}\right)^{2} \sup _{r \leq R_{\varepsilon, \lambda, \gamma}} r^{4-\delta} \frac{r^{2}}{\left(1+r^{2}\right)^{2}} \\
& +c_{\kappa} \lambda\left(\frac{\varepsilon}{\tau}\right)^{4-q} \sup _{r \leq R_{\varepsilon, \lambda, \gamma}} r^{4-\delta} \frac{r^{q}}{\left(1+r^{2}\right)^{q}} .
\end{aligned}
$$

Taking into account that for $r$ very large we have $\left(1+r^{2}\right)^{-\beta} \sim r^{-2 \beta}$, we obtain

$$
\begin{aligned}
\sup _{r \leq R_{\varepsilon, \lambda, \gamma}} r^{4-\delta}|\Re(0)| & \leq c_{\kappa} \gamma \varepsilon^{2}+c_{\kappa} \gamma \varepsilon^{2} R_{\varepsilon, \lambda, \gamma}^{2-\delta}+c_{\kappa} \lambda \varepsilon^{4-q} R_{\varepsilon, \lambda, \gamma}^{4-\delta-q} \\
& \leq c_{\kappa} \gamma \varepsilon^{2}+c_{\kappa} \gamma \varepsilon^{\delta} r_{\varepsilon, \lambda, \gamma}^{2-\delta}+c_{\kappa} \lambda \varepsilon^{\delta} r_{\varepsilon, \lambda, \gamma}^{4-q-\delta} \\
& \leq c_{\kappa} \varepsilon^{\delta} r_{\varepsilon, \lambda, \gamma}^{2} .
\end{aligned}
$$

Recall that $\aleph(h):=\mathcal{G}_{\delta} \circ \mathcal{E}_{\delta} \circ \mathfrak{R}(h)$, then there exist $c_{\kappa}>0$ (which can depend only on $\kappa$ ), such that

$$
\|\aleph(0)\|_{C_{r a d, \delta}^{4, \alpha}\left(\mathbb{R}^{4}\right)} \leq c_{\kappa} \varepsilon^{\delta} r_{\varepsilon, \lambda, \gamma}^{2}
$$

Making use of Proposition 3.1 together with (28), hence there exist $\bar{c}_{\kappa}>0$ (which can depend only on $\kappa$ ), such that

$$
\|h\|_{\mathcal{C}_{r a d, \delta}^{4, \alpha}\left(\mathbb{R}^{4}\right)} \leq 2 \bar{c}_{\kappa} \varepsilon^{\delta} r_{\varepsilon, \lambda, \gamma}^{2} .
$$

Now, we recall an important result which play a center role in our estimates, see for example [23] and some references therein :

Lemma 3.2. [23] Given $x$ and $y$ two real numbers, $x>0, q \geq 1$ and for any small $\eta \in \mathbb{R}$ there exists a positive constant $C_{\eta}$ such that

$$
|| x+\left.y\right|^{q}-\left.x^{q}\left|\leq(1+\eta) q x^{q-1}\right| y\left|+C_{\eta}\right| y\right|^{q} .
$$

Now, let $h_{1}, h_{2}$ in $B\left(0,2 c \varepsilon^{\delta} r_{\varepsilon, \lambda, \gamma}^{2}\right)$ of $\mathcal{C}_{\text {rad, } \delta}^{4, \alpha}\left(\mathbb{R}^{4}\right)$, then given $\kappa>0$, there exist $c_{\kappa}>0$ (which can depend only on $\kappa$ ), such that for $\delta \in(0, \min (1,4-q)$ ), we 
SINGULAR LIMITS FOR 4-DIMENSIONAL GENERAL STATIONARY

have

$$
\begin{aligned}
& \sup _{r \leq R_{\varepsilon, \lambda, \gamma}} r^{4-\delta}\left|\mathfrak{R}\left(h_{2}\right)-\mathfrak{R}\left(h_{1}\right)\right| \\
& \leq c_{\kappa} \sup _{r \leq R_{\varepsilon, \lambda, \gamma}} r^{4-\delta}\left(1+|x|^{2}\right)^{-4}\left|e^{h_{2}}-e^{h_{1}}+h_{1}-h_{2}\right| \\
& +c_{\kappa} \lambda \varepsilon^{4-q} \sup _{r \leq R_{\varepsilon, \lambda, \gamma}} r^{4-\delta}\left(\left|\nabla\left(u_{1}+h_{2}\right)\right|^{q}-\left|\nabla\left(u_{1}+h_{1}\right)\right|^{q}\right) \\
& +c_{\kappa} \gamma \varepsilon^{2} \sup _{r \leq R_{\varepsilon, \lambda, \gamma}} r^{4-\delta}\left|\Delta\left(u_{1}+h_{2}\right)-\Delta\left(u_{1}+h_{1}\right)\right|
\end{aligned}
$$

Again, making use of Lemma 3.2 and recall that a functions $w$ in $\mathcal{C}_{r a d, \delta}^{k, \alpha}\left(\mathbb{R}^{4}\right)$ are bounded by a constant times $\left(1+r^{2}\right)^{\delta / 2}$ and have their $\ell-t h$ partial derivatives that are bounded by $\left(1+r^{2}\right)^{(\delta-\ell) / 2}$, for $\ell=1, \cdots, k+\alpha$ (a.e $\left|\nabla^{\ell} w\right| \leq c_{\kappa} r^{\delta-\ell}\|w\|_{\mathcal{C}_{\text {rad, } \delta(\mathbb{R})}^{4, \alpha}\left(\mathbb{R}^{4}\right)},\left(1+r^{2}\right)^{(\delta-\ell) / 2} \sim r^{\delta-\ell}$ for $r$ very large), then there exist $c_{\kappa}>0$ (only depend on $\kappa$ ) such that

$$
\begin{aligned}
& \sup _{r \leq R_{\varepsilon, \lambda, \gamma}} r^{4-\delta}\left|\mathfrak{R}\left(h_{2}\right)-\mathfrak{R}\left(h_{1}\right)\right| \\
& \leq c_{\kappa} \sup _{r \leq R_{\varepsilon, \lambda, \gamma}} r^{-4-\delta}\left|h_{2}-h_{1}\right|\left|h_{2}+h_{1}\right| \\
& +c_{\kappa} \lambda \varepsilon^{4-q} \sup _{r \leq R_{\varepsilon, \lambda, \gamma}} r^{4-\delta}\left[\left|\nabla\left(u_{1}+h_{1}\right)\right|^{q-1}+\left|\nabla\left(h_{2}-h_{1}\right)\right|^{q-1}\right]\left|\nabla\left(h_{2}-h_{1}\right)\right| \\
& +c_{\kappa} \gamma \varepsilon^{2} \sup _{r \leq R_{\varepsilon, \lambda, \gamma}} r^{4-\delta}\left|\Delta\left(h_{2}-h_{1}\right)\right| \\
& \leq c_{\kappa} \sum_{i=1}^{2}\left\|h_{i}\right\|_{\mathcal{C}_{r a d, \delta}^{4, \alpha}\left(\mathbb{R}^{4}\right)}\left\|h_{2}-h_{1}\right\|_{\mathcal{C}_{r a d, \delta}^{4, \alpha}\left(\mathbb{R}^{4}\right)}+c_{\kappa} \gamma \varepsilon^{2} R_{\varepsilon, \lambda, \gamma}^{2}\left\|h_{2}-h_{1}\right\|_{\mathcal{C}_{r a d, \delta}^{4, \alpha}\left(\mathbb{R}^{4}\right)} \\
& +c_{\kappa} \lambda \varepsilon^{4-q} \sup _{r \leq R_{\varepsilon, \lambda, \gamma}} r^{4-\delta}\left[\left|\nabla u_{1}\right|^{q-1}+\left|\nabla h_{1}\right|^{q-1}+\left|\nabla h_{2}\right|^{q-1}\right]\left|\nabla\left(h_{2}-h_{1}\right)\right| \\
& \leq c_{\kappa} \sum_{i=1}^{2}\left\|h_{i}\right\|_{\mathcal{C}_{r a d, \delta}^{4, \alpha}\left(\mathbb{R}^{4}\right)}\left\|h_{2}-h_{1}\right\|_{\mathcal{C}_{r a d, \delta}^{4, \alpha}\left(\mathbb{R}^{4}\right)}+c_{\kappa} \gamma \varepsilon^{2} R_{\varepsilon, \lambda, \gamma}^{2}\left\|h_{2}-h_{1}\right\|_{\mathcal{C}_{r a d, \delta}^{4, \alpha}\left(\mathbb{R}^{4}\right)} \\
& \left.+c_{\kappa} \lambda \varepsilon^{4-q}\left[R_{\varepsilon, \lambda, \gamma}^{4-q}+R_{\varepsilon, \lambda, \gamma}^{4+\delta q-\delta-q} \sum_{i=1}^{2}\left\|h_{i}\right\|_{\mathcal{C}_{r a d, \delta}^{4, \alpha}}^{q-1} \mathbb{R}^{4}\right)\right]\left\|h_{2}-h_{1}\right\|_{\mathcal{C}_{r a d, \delta}^{4, \alpha}\left(\mathbb{R}^{4}\right)} .
\end{aligned}
$$

Provided $h_{i} \in \mathcal{C}_{r a d, \delta}^{4, \alpha}\left(\mathbb{R}^{4}\right)$ satisfies $\left\|h_{i}\right\|_{\mathcal{C}_{r a d, \delta}^{4, \alpha}\left(\mathbb{R}^{4}\right)} \leq 2 c_{\kappa} \varepsilon^{\delta} r_{\varepsilon, \lambda, \gamma}^{2}$, then the last estimate, is given by

$$
\begin{aligned}
\sup _{r \leq R_{\varepsilon, \lambda, \gamma} r^{4-\delta}\left|\mathfrak{R}\left(h_{2}\right)-\mathfrak{R}\left(h_{1}\right)\right| \leq} & c_{\kappa} \varepsilon^{\delta} r_{\varepsilon, \lambda, \gamma}^{2}\left\|h_{2}-h_{1}\right\|_{\mathfrak{e}_{r a d, \delta}^{4, \alpha}\left(\mathbb{R}^{4}\right)}+c_{\kappa} \gamma r_{\varepsilon, \lambda, \gamma}^{2}\left\|h_{2}-h_{1}\right\|_{\mathcal{C}_{r a d, \delta}^{4, \alpha}\left(\mathbb{R}^{4}\right)} \\
& +c_{\kappa} \lambda\left[r_{\varepsilon, \lambda, \gamma}^{4-q}+r_{\varepsilon, \lambda, \gamma}^{2+\delta(q-1)+q}\right]\left\|h_{2}-h_{1}\right\|_{\mathcal{E}_{r a d, \delta}^{4, \alpha}\left(\mathbb{R}^{4}\right)} .
\end{aligned}
$$

Similarly, making use of Proposition 3.1 together with (28), we conclude that given $\kappa>0$, there exist $\varepsilon_{\kappa}, \lambda_{\kappa}, \gamma_{\kappa}$ and $\bar{c}_{\kappa}>0$ (only depend on $\kappa$ ) such that

$$
\left\|\aleph\left(h_{2}\right)-\aleph\left(h_{1}\right)\right\|_{\mathcal{C}_{r a d, \delta}^{4, \alpha}\left(\mathbb{R}^{4}\right)} \leq \bar{c}_{\kappa} r_{\varepsilon, \lambda, \gamma}^{2}\left\|h_{2}-h_{1}\right\|_{\mathcal{C}_{r a d, \delta}^{4, \alpha}\left(\mathbb{R}^{4}\right)}
$$


SINGULAR LIMITS FOR 4-DIMENSIONAL GENERAL STATIONARY

Q-KURAMOTO-SIVASHINSKY EQUATION (Q-KSE) WITH EXPONENTIAL

Reducing $\varepsilon_{\kappa}, \lambda_{\kappa}$ and $\gamma_{\kappa}$ if necessary, we can assume that,

$$
\bar{c}_{\kappa} r_{\varepsilon, \lambda, \gamma}^{2} \leq \frac{1}{2}
$$

for all $\varepsilon \in\left(0, \varepsilon_{\kappa}\right), \lambda \in\left(0, \lambda_{\kappa}\right)$ and $\gamma \in\left(0, \gamma_{\kappa}\right)$. Then, (31) and (32) are enough to show that

$$
h \longmapsto \aleph(h)
$$

is a contraction from the ball

$$
\left\{h \in \mathcal{C}_{\text {rad }, \delta}^{4, \alpha}\left(\mathbb{R}^{4}\right):\|h\|_{\mathcal{C}_{\text {rad, }}^{4, \alpha}\left(\mathbb{R}^{4}\right)} \leq 2 c_{\kappa} \varepsilon^{\delta} r_{\varepsilon, \lambda, \gamma}^{2}\right\}
$$

into itself and hence has a unique fixed point $h$ in this set. This fixed point is a solution of $(29)$ in $\bar{B}_{R_{\varepsilon, \lambda, \gamma}}$.

We summarize this in the following proposition.

Proposition 3.2. Let $q \in[1,4)$ and $\delta \in(0, \min (1,4-q))$. Given $\kappa>0$, there exist $\varepsilon_{\kappa}>0, \lambda_{\kappa}>0, \gamma_{\kappa}>0$ and $c_{\kappa}>0$ (which can depend only on $\kappa$ ) such that for all for all $\varepsilon \in\left(0, \varepsilon_{\kappa}\right), \lambda \in\left(0, \lambda_{\kappa}\right)$ and $\gamma \in\left(0, \gamma_{\kappa}\right)$, there exists a unique solution $h \in \mathrm{C}_{\text {rad, }, \delta}^{4, \alpha}\left(\mathbb{R}^{4}\right)$ of (29) such that

$$
v(x)=u_{1}(x)+h(x)
$$

solves (25) in $\bar{B}_{R_{\varepsilon, \lambda, \gamma}}$. In addition

$$
\|h\|_{\mathcal{C}_{\text {rad, }}^{4, \alpha}\left(\mathbb{R}^{4}\right)} \leq 2 c_{\kappa} \varepsilon^{\delta} r_{\varepsilon, \lambda, \gamma}^{2}
$$

* For $q=4$

Recall that

$$
\sup _{r \leq R_{\varepsilon, \lambda, \gamma}} r^{4-\delta}|\Re(0)| \leq c_{\kappa} \gamma\left(\frac{\varepsilon}{\tau}\right)^{2} \sup _{r \leq R_{\varepsilon, \lambda, \gamma}} r^{4-\delta} \Delta u_{1}+c_{\kappa} \lambda \sup _{r \leq R_{\varepsilon, \lambda, \gamma}} r^{4-\delta}\left|\nabla u_{1}\right|^{4} .
$$

Since

$$
\lambda \sup _{r \leq R_{\varepsilon, \lambda, \gamma}} r^{4-\delta}\left|\nabla u_{1}\right|^{4}=\lambda \sup _{r \leq R_{\varepsilon, \lambda, \gamma}} r^{4-\delta} \frac{r^{4}}{\left(1+r^{2}\right)^{4}}
$$

taking into account that for $r$ very large, we have $\left(1+r^{2}\right)^{-4} \sim r^{-8}$ then, there exist $c_{\kappa}>0$ (which can depend only on $\kappa$ ), such that 
SINGULAR LIMITS FOR 4-DIMENSIONAL GENERAL STATIONARY

$$
\begin{aligned}
\sup _{r \leq R_{\varepsilon, \lambda, \gamma}} r^{4-\delta}|\mathfrak{R}(0)| & \leq c_{\kappa} \gamma \varepsilon^{2}+c_{\kappa} \gamma \varepsilon^{\delta} r_{\varepsilon, \lambda, \gamma}^{2-\delta}+c_{\kappa} \lambda \\
& \leq c_{\kappa} r_{\varepsilon, \lambda, \gamma}^{2} .
\end{aligned}
$$

Then there exist $c_{\kappa}>0$ (which can depend only on $\kappa$ ), such that

$$
\|\aleph(0)\|_{C_{\text {rad, }, \delta}^{4, \alpha}\left(\mathbb{R}^{4}\right)} \leq c_{\kappa} r_{\varepsilon, \lambda, \gamma}^{2} .
$$

Making use of Proposition 3.1 together with (28), we conclude that there exists a unique solution $\hbar \in \mathcal{C}_{\text {rad, }, \delta}^{4, \alpha}\left(\mathbb{R}^{4}\right)$ of (29) such that

$$
\|\hbar\|_{\mathcal{C}_{\text {rad, }}^{4, \alpha}\left(\mathbb{R}^{4}\right)} \leq 2 c_{\kappa} r_{\varepsilon, \lambda, \gamma}^{2} .
$$

Let, let $\hbar_{1}, \hbar_{2}$ in $B\left(0,2 c_{\kappa} r_{\varepsilon, \lambda, \gamma}^{2}\right)$ of $\mathcal{C}_{r a d, \delta}^{4, \alpha}\left(\mathbb{R}^{4}\right)$, satisfying for each $x \in$ $\bar{B}_{R_{\varepsilon, \lambda, \gamma}}$

$$
\left|\hbar_{i}(x)\right| \leq c_{\kappa} r_{\varepsilon, \lambda, \gamma}^{2+\delta} \varepsilon^{-\delta} \leq\left\{\begin{array}{rll}
c_{\kappa} \varepsilon^{1-\delta / 2} & \text { for } & \varepsilon \geq \max (\lambda, \gamma) \\
c_{\kappa} \lambda^{1+\delta / 2} \varepsilon^{-\delta} & \text { for } & \lambda>\max (\varepsilon, \gamma) \\
c_{\kappa} \gamma^{1+\delta / 2} \varepsilon^{-\delta} & \text { for } & \gamma>\max (\varepsilon, \lambda)
\end{array}\right.
$$

then using condition $\left(A_{\varepsilon, \lambda, \gamma}\right)$, we prove that $|\hbar(x)| \longrightarrow 0$ as $\varepsilon, \lambda$ and $\gamma$ tend to 0 .

Now, given $\kappa>0$, there exist $c_{\kappa}>0$ (which can depend only on $\kappa$ ), such that for $\delta \in(0,1)$, we have

$$
\begin{aligned}
& \sup _{r \leq R_{\varepsilon, \lambda, \gamma}} r^{4-\delta}\left|\mathfrak{R}\left(\hbar_{2}\right)-\mathfrak{R}\left(\hbar_{1}\right)\right| \\
& \leq c_{\kappa} \sup _{r \leq R_{\varepsilon, \lambda, \gamma}} r^{4-\delta}\left(1+|x|^{2}\right)^{-4}\left|e^{\hbar_{2}}-e^{\hbar_{1}}+\hbar_{1}-\hbar_{2}\right| \\
& +c_{\kappa} \lambda \sup _{r \leq R_{\varepsilon, \lambda, \gamma}} r^{4-\delta}\left(\left|\nabla\left(u_{1}+\hbar_{2}\right)\right|^{4}-\left|\nabla\left(u_{1}+\hbar_{1}\right)\right|^{4}\right) \\
& +c_{\kappa} \gamma \varepsilon^{2} \sup _{r \leq R_{\varepsilon, \lambda, \gamma}} r^{4-\delta}\left|\Delta\left(u_{1}+\hbar_{2}\right)-\Delta\left(u_{1}+\hbar_{1}\right)\right|
\end{aligned}
$$

Again, making use of Lemma 3.2 for $q=4$ and recall that a functions $w$ in $\mathrm{C}_{r a d, \delta}^{k, \alpha}\left(\mathbb{R}^{4}\right)$ are bounded by a constant times $\left(1+r^{2}\right)^{\delta / 2}$ and have their $\ell-t h$ partial derivatives that are bounded by $\left(1+r^{2}\right)^{(\delta-\ell) / 2}$, for $\ell=1, \cdots, k+\alpha$ (a.e $\left|\nabla^{\ell} w\right| \leq c_{\kappa} r^{\delta-\ell}\|w\|_{\mathcal{C}_{\text {rad, }, \delta}^{4, \alpha}\left(\mathbb{R}^{4}\right)},\left(1+r^{2}\right)^{(\delta-\ell) / 2} \sim r^{\delta-\ell}$ for $r$ very large), then 
SINGULAR LIMITS FOR 4-DIMENSIONAL GENERAL STATIONARY

Q-KURAMOTO-SIVASHINSKY EQUATION (Q-KSE) WITH EXPONENTIAL

there exist $c_{\kappa}>0$ (only depend on $\kappa$ ) such that

$$
\begin{aligned}
& \sup _{r \leq R_{\varepsilon, \lambda, \gamma}} r^{4-\delta}\left|\mathfrak{R}\left(\hbar_{2}\right)-\mathfrak{R}\left(\hbar_{1}\right)\right| \\
& \leq c_{\kappa} \sup _{r \leq R_{\varepsilon, \lambda, \gamma}} r^{-4-\delta}\left|\hbar_{2}-\hbar_{1}\right|\left|\hbar_{2}+\hbar_{1}\right| \\
& +c_{\kappa} \lambda \sup _{r \leq R_{\varepsilon, \lambda, \gamma}} r^{4-\delta}\left[\left|\nabla\left(u_{1}+\hbar_{1}\right)\right|^{3}+\left|\nabla\left(\hbar_{2}-\hbar_{1}\right)\right|^{3}\right]\left|\nabla\left(\hbar_{2}-\hbar_{1}\right)\right| \\
& +c_{\kappa} \gamma \varepsilon^{2} \sup _{r \leq R_{\varepsilon, \lambda, \gamma}} r^{4-\delta}\left|\Delta\left(\hbar_{2}-\hbar_{1}\right)\right|
\end{aligned}
$$

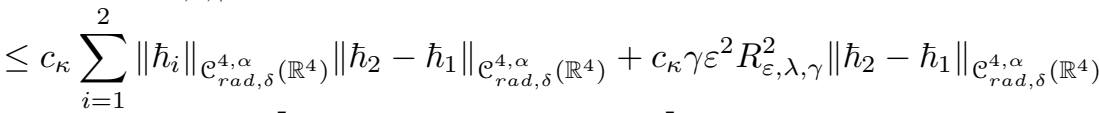

$$
\begin{aligned}
& +c_{\kappa} \lambda \sup _{r \leq R_{\varepsilon, \lambda, \gamma}} r^{4-\delta}\left[\left|\nabla u_{1}\right|^{3}+\left|\nabla \hbar_{1}\right|^{3}+\left|\nabla \hbar_{2}\right|^{3}\right]\left|\nabla\left(\hbar_{2}-\hbar_{1}\right)\right|
\end{aligned}
$$

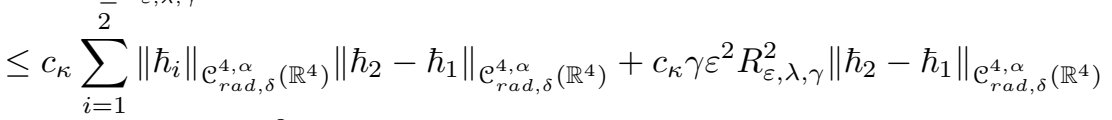

$$
\begin{aligned}
& +c_{\kappa} \lambda\left[1+R_{\varepsilon, \lambda, \gamma}^{3 \delta} \sum_{i=1}^{2}\left\|\hbar_{i}\right\|_{\mathcal{C}_{\text {rad }, \delta}^{4, \alpha}\left(\mathbb{R}^{4}\right)}^{3}\right]\left\|\hbar_{2}-\hbar_{1}\right\|_{\mathcal{C}_{\text {rad, }}^{4, \alpha}\left(\mathbb{R}^{4}\right)} .
\end{aligned}
$$

Provided $\hbar_{i} \in \mathcal{C}_{r a d, \delta}^{4, \alpha}\left(\mathbb{R}^{4}\right)$ satisfies $\left\|\hbar_{i}\right\|_{\mathcal{E}_{r a d, \delta}^{4, \alpha}\left(\mathbb{R}^{4}\right)} \leq 2 c_{\kappa} r_{\varepsilon, \lambda, \gamma}^{2}$, then the last estimate, is given by

$$
\begin{aligned}
& \sup _{r \leq R_{\varepsilon, \lambda, \gamma}} r^{4-\delta}\left|\mathfrak{R}\left(\hbar_{2}\right)-\mathfrak{R}\left(\hbar_{1}\right)\right| \leq c_{\kappa} r_{\varepsilon, \lambda, \gamma}^{2}\left\|\hbar_{2}-\hbar_{1}\right\|_{\mathcal{C}_{\text {rad, }}^{4, \alpha}\left(\mathbb{R}^{4}\right)}+c_{\kappa} \gamma r_{\varepsilon, \lambda, \gamma}^{2}\left\|\hbar_{2}-\hbar_{1}\right\|_{\mathcal{C}_{\text {rad, }}^{4, \alpha}\left(\mathbb{R}^{4}\right)} \\
& +c_{\kappa} \lambda\left[1+\left(r_{\varepsilon, \lambda, \gamma}^{2+\delta} \varepsilon^{-\delta}\right)^{3}\right]\left\|\hbar_{2}-\hbar_{1}\right\|_{\mathcal{C}_{\text {rad, }}^{4, \alpha}\left(\mathbb{R}^{4}\right)} .
\end{aligned}
$$

Similarly, making use of Proposition 3.1 together with (28), using the condition $\left(A_{\varepsilon, \lambda, \gamma}\right)$ we conclude that given $\kappa>0$, there exist $\varepsilon_{\kappa}, \lambda_{\kappa}, \gamma_{\kappa}$ and $\bar{c}_{\kappa}>0$ (only depend on $\kappa$ ) such that

$$
\left\|\aleph\left(\hbar_{2}\right)-\aleph\left(\hbar_{1}\right)\right\|_{\mathcal{C}_{\text {rad, }}^{4, \alpha}\left(\mathbb{R}^{4}\right)} \leq \bar{c}_{\kappa} r_{\varepsilon, \lambda, \gamma}^{2}\left\|\hbar_{2}-\hbar_{1}\right\|_{\mathcal{C}_{r a d, \delta}^{4, \alpha}\left(\mathbb{R}^{4}\right)} .
$$

Reducing $\varepsilon_{\kappa}, \lambda_{\kappa}$ and $\gamma_{\kappa}$ if necessary, we can assume that,

$$
\bar{c}_{\kappa} r_{\varepsilon, \lambda, \gamma}^{2} \leq \frac{1}{2}
$$

for all $\varepsilon \in\left(0, \varepsilon_{\kappa}\right), \lambda \in\left(0, \lambda_{\kappa}\right)$ and $\gamma \in\left(0, \gamma_{\kappa}\right)$ satisfying $\left(A_{\varepsilon, \lambda, \gamma}\right)$. Then, (33) and (34) are enough to show that

$$
\hbar \longmapsto \aleph(\hbar)
$$

is a contraction from the ball

$$
\left\{\hbar \in \mathcal{C}_{r a d, \delta}^{4, \alpha}\left(\mathbb{R}^{4}\right):\|\hbar\|_{\mathcal{C}_{\text {rad, }}^{4, \alpha}\left(\mathbb{R}^{4}\right)} \leq 2 c_{\kappa} r_{\varepsilon, \lambda, \gamma}^{2}\right\}
$$


SINGULAR LIMITS FOR 4-DIMENSIONAL GENERAL STATIONARY

into itself and hence has a unique fixed point $\hbar$ in this set. This fixed point is a solution of (29) in $\bar{B}_{R_{\varepsilon, \lambda, \gamma}}$.

We summarize this in the following proposition.

Proposition 3.3. Let $q=4$ and $\delta \in(0,1)$. Given $\kappa>0$, there exist $\varepsilon_{\kappa}>0$, $\lambda_{\kappa}>0, \gamma_{\kappa}>0$ (which can depend only on $\kappa$ ) and $c>0$ such that for all for all $\varepsilon \in\left(0, \varepsilon_{\kappa}\right), \lambda \in\left(0, \lambda_{\kappa}\right)$ and $\gamma \in\left(0, \gamma_{\kappa}\right)$ satisfying $\left(A_{\varepsilon, \lambda, \gamma}\right)$, there exists a unique solution $\hbar \in \mathcal{C}_{\text {rad, }, \delta}^{4, \alpha}\left(\mathbb{R}^{4}\right)$ of (29) such that

$$
v(x)=u_{1}(x)+\hbar(x)
$$

solves (25) in $\bar{B}_{R_{\varepsilon, \lambda, \gamma}}$. In addition

$$
\|\hbar\|_{\mathcal{C}_{r a d, \delta}^{4, \alpha}\left(\mathbb{R}^{4}\right)} \leq 2 c_{\kappa} r_{\varepsilon, \lambda, \gamma}^{2}
$$

\section{Known results [1]}

\subsection{Analysis of the bi-Laplace operator in weighted spaces}

Given $x^{1}, \ldots, x^{m} \in \Omega$ we define $X:=\left(x^{1}, \ldots, x^{m}\right)$ and

$$
\bar{\Omega}^{*}(X):=\bar{\Omega}-\left\{x^{1}, \ldots, x^{m}\right\},
$$

and we choose $r_{0}>0$ so that the balls $B_{r_{0}}\left(x^{i}\right)$ of center $x^{i}$ and radius $r_{0}$ are mutually disjoint and included in $\Omega$. For all $r \in\left(0, r_{0}\right)$ we define

$$
\bar{\Omega}_{r}(X):=\bar{\Omega}-\cup_{j=1}^{m} B_{r}\left(x^{j}\right)
$$

With these notations, we have the :

Definition 4.1. Given $k \in \mathbb{R}, \alpha \in(0,1)$ and $\nu \in \mathbb{R}$, we introduce the Hölder weighted space $\mathrm{C}_{\nu}^{k, \alpha}\left(\bar{\Omega}^{*}(X)\right)$ as the space of functions $w \in \mathcal{C}_{l o c}^{k, \alpha}\left(\bar{\Omega}^{*}(X)\right)$ which is endowed with the norm

$\|w\|_{\mathfrak{C}_{\nu}^{k, \alpha}\left(\bar{\Omega}^{*}(X)\right)}:=\|w\|_{\mathfrak{e}^{k, \alpha}\left(\bar{\Omega}_{r_{0} / 2}(X)\right)}+\sum_{j=1}^{m} \sup _{r \in\left(0, r_{0} / 2\right)}\left(r^{-\nu}\left\|w\left(x^{j}+r \cdot\right)\right\|_{\mathfrak{e}^{k, \alpha}\left(\bar{B}_{2}-B_{1}\right)}\right)$,

is finite.

Again, these spaces have already been used many times in nonlinear contexts and we refer to [16]. Roughly speaking, functions in $\mathcal{C}_{\nu}^{k, \alpha}\left(\bar{\Omega}^{*}(X)\right)$ re bounded by a constant times the distance to $X$ to the power $\nu$ and have their $\ell-t h$ partial derivatives that are bounded by a constant times the distance 
SINGULAR LIMITS FOR 4-DIMENSIONAL GENERAL STATIONARY

Q-KURAMOTO-SIVASHINSKY EQUATION (Q-KSE) WITH EXPONENTIAL

NONLINEARITY

to $X$ to the power $\nu-\ell$, for $\ell=1, \cdots, k+\alpha$.

When $k \geq 2$, we denote by $\left[\mathcal{C}_{\nu}^{k, \alpha}\left(\bar{\Omega}^{*}(X)\right)\right]_{0}$ be the subspace of functions $w \in \mathcal{C}_{\nu}^{k, \alpha}\left(\bar{\Omega}^{*}(X)\right)$ satisfying $w=\Delta w=0$ on $\partial \Omega$.

We will use the following :

Proposition 4.1. [1] Assume that $\nu<0$ and $\nu \notin \mathbb{Z}$, then

$$
\begin{array}{ccc}
\mathcal{L}_{\nu}:\left[\mathcal{C}_{\nu}^{4, \alpha}\left(\bar{\Omega}^{*}(X)\right)\right]_{0} & \longrightarrow & \mathcal{C}_{\nu-4}^{0, \alpha}\left(\bar{\Omega}^{*}(X)\right) \\
w & \longmapsto & \Delta^{2} w
\end{array}
$$

is surjective.

\subsection{Bi-harmonic extensions}

Given $\varphi \in \mathcal{C}^{4, \alpha}\left(S^{3}\right)$ and $\psi \in \mathcal{C}^{2, \alpha}\left(S^{3}\right)$ we define $H^{i}\left(=H^{i}(\varphi, \psi ; \cdot)\right)$ to be the solution of

$$
\left\{\begin{aligned}
\Delta^{2} H^{i} & =0 \text { in } B_{1} \\
H^{i} & =\varphi \text { on } \partial B_{1} \\
\Delta H^{i} & =\psi \text { on } \partial B_{1},
\end{aligned}\right.
$$

where, as already mentioned, $B_{1}$ denotes the unit ball in $\mathbb{R}^{4}$.

We set $B_{1}^{*}=B_{1}-\{0\}$. As in the previous section, we define :

Definition 4.2. Given $k \in \mathbb{N}, \alpha \in(0,1)$ and $\mu \in \mathbb{R}$, we introduce the Hölder weighted spaces $\mathcal{C}_{\mu}^{k, \alpha}\left(\bar{B}_{1}^{*}\right)$ as the space of function in $\mathcal{C}_{\text {loc }}^{k, \alpha}\left(\bar{B}_{1}^{*}\right)$ for which the following norm

$$
\|u\|_{\mathcal{C}_{\mu}^{k, \alpha}\left(\bar{B}_{1}^{*}\right)}=\sup _{r \leq 1 / 2}\left(r^{-\mu}\|u(r \cdot)\|_{\mathcal{C}^{k, \alpha}\left(\bar{B}_{2}-B_{1}\right)}\right)
$$

is finite.

This corresponds to the space and norm already defined in the previous section when $\Omega=B_{1}, m=1$ and $x^{1}=0$.

Let $e_{1}, \ldots, e_{4}$ be the coordinate functions on $S^{3}$. In [1] is proved that :

Lemma 4.1. [1] Assume that

$$
\int_{S^{3}}(8 \varphi-\psi) d v_{S^{3}}=0 \quad \text { and also that } \quad \int_{S^{3}}(12 \varphi-\psi) e_{\ell} d v_{S^{3}}=0
$$


SINGULAR LIMITS FOR 4-DIMENSIONAL GENERAL STATIONARY

Q-KURAMOTO-SIVASHINSKY EQUATION (Q-KSE) WITH EXPONENTIAL

NONLINEARITY

for $\ell=1, \ldots, 4$. Then there exists $c>0$ such that

$$
\left\|H^{i}(\varphi, \psi ; \cdot)\right\|_{\mathcal{C}_{2}^{4, \alpha}\left(\bar{B}_{1}^{*}\right)} \leq c\left(\|\varphi\|_{\mathcal{C}^{4, \alpha}\left(S^{3}\right)}+\|\psi\|_{\mathcal{C}^{2, \alpha}\left(S^{3}\right)}\right) .
$$

Observe that, under the hypothesis (36), the coefficients of $r^{0}$ and $r^{1}$ vanish and hence, at least formally, the expansion of $H^{i}$ only involves powers of $r$ that are greater than or equal to 2 .

Given $\varphi \in \mathcal{C}^{4, \alpha}\left(S^{3}\right)$ and $\psi \in \mathcal{C}^{2, \alpha}\left(S^{3}\right)$ we define (when it exists !) $H^{e}(=$ $\left.H^{e}(\varphi, \psi ; \cdot)\right)$ to be the solution of

$$
\left\{\begin{aligned}
\Delta^{2} H^{e} & =0 \text { in } \mathbb{R}^{4}-B_{1} \\
H^{e} & =\varphi \text { on } \partial B_{1} \\
\Delta H^{e} & =\psi \text { on } \partial B_{1},
\end{aligned}\right.
$$

which decays at infinity.

Definition 4.3. Given $k \in \mathbb{N}, \alpha \in(0,1)$ and $\nu \in \mathbb{R}$, we define the space $\mathcal{C}_{\nu}^{k, \alpha}\left(\mathbb{R}^{4}-B_{1}\right)$ as the space of functions $w \in \mathcal{C}_{l o c}^{k, \alpha}\left(\mathbb{R}^{4}-B_{1}\right)$ for which the following norm

$$
\|w\|_{\mathcal{C}_{\nu}^{k, \alpha}\left(\mathbb{R}^{4}-B_{1}\right)}=\sup _{r \geq 1}\left(r^{-\nu}\|w(r \cdot)\|_{\mathcal{C}_{\nu}^{k, \alpha}\left(\bar{B}_{2}-B_{1}\right)}\right),
$$

is finite.

We recall the :

Lemma 4.2. [1] Assume that

$$
\int_{S^{3}} \psi d v_{S^{3}}=0
$$

Then there exists $c>0$ such that

$$
\left\|H^{e}(\varphi, \psi ; \cdot)\right\|_{\mathcal{C}_{-1}^{4, \alpha}\left(\mathbb{R}^{4}-B_{1}\right)} \leq c\left(\|\varphi\|_{\mathcal{C}^{4, \alpha}\left(S^{3}\right)}+\|\psi\|_{\mathcal{C}^{2, \alpha}\left(S^{3}\right)}\right) .
$$

Observe that (38) implies that the expansion of $H^{e}$ only involves powers of $r$ that are lower than or equal to -1 .

We will need the : 
SINGULAR LIMITS FOR 4-DIMENSIONAL GENERAL STATIONARY

Q-KURAMOTO-SIVASHINSKY EQUATION (Q-KSE) WITH EXPONENTIAL

Lemma 4.3. [1] The mapping

$$
\begin{aligned}
\mathcal{P}: \quad \mathcal{C}^{4, \alpha}\left(S^{3}\right)^{\perp} \times \mathcal{C}^{4, \alpha}\left(S^{3}\right)^{\perp} & \longrightarrow \quad \mathcal{C}^{3, \alpha}\left(S^{3}\right)^{\perp} \times \mathcal{C}^{1, \alpha}\left(S^{3}\right)^{\perp} \\
(\varphi, \psi) & \longmapsto\left(\partial_{r} H^{i}-\partial_{r} H^{e}, \partial_{r} \Delta H^{i}-\partial_{r} \Delta H^{e}\right)
\end{aligned}
$$

where $H^{i}=H^{i}(\varphi, \psi ; \cdot):=H_{\varphi, \psi}^{i}(\cdot)$ and $H^{e}=H^{e}(\varphi, \psi ; \cdot):=H_{\varphi, \psi}^{e}(\cdot)$, is an isomorphism.

\section{The first nonlinear Dirichlet problem}

Recall that for all $\varepsilon, \tau, \lambda, \gamma>0$, we have previously defined

$$
R_{\varepsilon, \lambda, \gamma}:=\tau r_{\varepsilon, \lambda, \gamma} / \varepsilon
$$

where

$$
r_{\varepsilon, \lambda, \gamma}:=\max (\sqrt{\varepsilon}, \sqrt{\lambda}, \sqrt{\gamma})
$$

and

$$
q \in[1,4]
$$

Given $\varphi \in \mathcal{C}^{4, \alpha}\left(S^{3}\right)$ and $\psi \in \mathcal{C}^{2, \alpha}\left(S^{3}\right)$ satisfying (36), we define

$$
\mathbf{u}:=u_{1}+h+H^{i}\left(\varphi, \psi ;\left(\cdot / R_{\varepsilon, \lambda, \gamma}\right)\right)
$$

where, for $q \in[1,4)$ and $\delta \in(0, \min (1,4-q))$,

$$
\|h\|_{\mathcal{C}_{\text {rad, }}^{4, \alpha}\left(\mathbb{R}^{4}\right)} \leq 2 c_{\kappa} \varepsilon^{\delta} r_{\varepsilon, \lambda, \gamma}^{2}
$$

and for $q=4, h:=\hbar$ and $\delta \in(0,1)$,

$$
\|\hbar\|_{\mathcal{C}_{\text {rad, }}^{4, \alpha}\left(\mathbb{R}^{4}\right)} \leq 2 c_{\kappa} r_{\varepsilon, \lambda, \gamma}^{2} .
$$

We would like to find a solution $u$ of

$$
\Delta^{2} u-\gamma\left(\frac{\varepsilon}{\tau}\right)^{2} \Delta u-\lambda\left(\frac{\varepsilon}{\tau}\right)^{4-q}|\nabla u|^{q}-24 e^{u}=0
$$

which is defined in $B_{R_{\varepsilon, \lambda, \gamma}}$ and which is a perturbation of $\mathbf{u}$. Writing $u=\mathbf{u}+v$, this amounts to solve the equation

$$
\begin{gathered}
\mathbb{L} v=\frac{384}{\left(1+r^{2}\right)^{4}} e^{h}\left(e^{H^{i}\left(\varphi, \psi ;\left(\cdot / R_{\varepsilon, \lambda, \gamma}\right)\right)+v}-1-v\right)+\frac{384}{\left(1+r^{2}\right)^{4}}\left(e^{h}-1\right) v \\
+\gamma\left(\frac{\varepsilon}{\tau}\right)^{2} \Delta\left(u_{1}+h+H^{i}\left(\varphi, \psi ;\left(\cdot / R_{\varepsilon, \lambda, \gamma}\right)\right)+v\right)-\gamma\left(\frac{\varepsilon}{\tau}\right)^{2} \Delta\left(u_{1}+h\right) \\
+\lambda\left(\frac{\varepsilon}{\tau}\right)^{4-q}\left|\nabla\left(u_{1}+h+H^{i}\left(\varphi, \psi ;\left(\cdot / R_{\varepsilon, \lambda, \gamma}\right)\right)+v\right)\right|^{q}-\lambda\left(\frac{\varepsilon}{\tau}\right)^{4-q}\left|\nabla\left(u_{1}+h\right)\right|^{q},
\end{gathered}
$$


SINGULAR LIMITS FOR 4-DIMENSIONAL GENERAL STATIONARY

Q-KURAMOTO-SIVASHINSKY EQUATION (Q-KSE) WITH EXPONENTIAL

NONLINEARITY

since $H^{i}$ is bi-harmonic. In the following, we will denote by $\mathcal{K}(v)$ the right hand side of (40).

We fix

$$
\mu \in(1,2)
$$

and denote by $\mathcal{G}_{\mu}$ a right inverse provided by Proposition 3.1. To find $\underline{\mathrm{a}}$ solution of (40), it is enough to find $v \in \mathcal{C}_{\mu}^{4, \alpha}\left(\mathbb{R}^{4}\right)$ solution of

$$
v=N(\varepsilon, \lambda, \gamma, \tau, \varphi, \psi ; v)
$$

where we have defined

$$
N(\varepsilon, \lambda, \gamma, \tau, \varphi, \psi ; v):=\mathcal{G}_{\mu} \circ \mathcal{E}_{R_{\varepsilon, \lambda, \gamma}}(\mathcal{K}(v))
$$

Given $\kappa>1$ (whose value will be fixed later on), we now further assume that the functions $\varphi \in \mathfrak{C}^{4, \alpha}\left(S^{3}\right), \psi \in \mathfrak{C}^{2, \alpha}\left(S^{3}\right)$ and the constant $\tau>0$ satisfy $\frac{1}{\log 1 / r_{\varepsilon, \lambda, \gamma}^{2}}\left|\log \left(\tau / \tau_{*}\right)\right| \leq \kappa r_{\varepsilon, \lambda, \gamma}^{2},\|\varphi\|_{\mathcal{e}^{4, \alpha}\left(S^{3}\right)} \leq \kappa r_{\varepsilon, \lambda, \gamma}^{2}$ and $\|\psi\|_{\mathrm{e}^{2, \alpha}\left(S^{3}\right)} \leq \kappa r_{\varepsilon, \lambda, \gamma}^{2}$, where $\tau_{*}>0$ is fixed later.

We have the following technical :

Lemma 5.1. Let $q \in[1,4]$. Given $\kappa>0, \mu \in(1,2)$ and $\delta \in(0, \min (1, q-4))$, there exist $\varepsilon_{\kappa}>0, \lambda_{\kappa}>0, \gamma_{\kappa}>0, c_{\kappa}>0$ and $\bar{c}_{\kappa}>0$ such that, for all $\varepsilon \in\left(0, \varepsilon_{\kappa}\right), \lambda \in\left(0, \lambda_{\kappa}\right)$ and $\gamma \in\left(0, \gamma_{\kappa}\right)$ (satisfying the condition $\left(A_{\varepsilon, \lambda, \gamma}\right)$ in case $q=4)$, then

$$
\|N(\varepsilon, \lambda, \gamma, \tau, \varphi, \psi ; 0)\|_{\mathcal{C}_{\mu}^{4, \alpha}\left(\mathbb{R}^{4}\right)} \leq c_{\kappa} \varepsilon^{\mu} r_{\varepsilon, \lambda, \gamma}^{2}
$$

Moreover,

$$
\begin{aligned}
& \left\|N\left(\varepsilon, \lambda, \gamma, \tau, \varphi, \psi ; v_{2}\right)-N\left(\varepsilon, \lambda, \gamma, \tau, \varphi, \psi ; v_{1}\right)\right\|_{\mathcal{C}_{\mu}^{4, \alpha}\left(\mathbb{R}^{4}\right)} \leq \bar{c}_{\kappa} r_{\varepsilon, \lambda, \gamma}^{2}\left\|v_{2}-v_{1}\right\|_{\mathcal{C}_{\mu}^{4, \alpha}\left(\mathbb{R}^{4}\right)} \\
& \quad \text { provided } \tilde{v}=v_{1}, v_{2} \in \mathcal{C}_{\mu}^{4, \alpha}\left(\mathbb{R}^{4}\right), \varphi \in \mathcal{C}^{4, \alpha}\left(S^{3}\right), \psi \in \mathcal{C}^{4, \alpha}\left(S^{3}\right) \text { satisfy } \\
& \|\tilde{v}\|_{\mathcal{C}_{\mu}^{4, \alpha}\left(\mathbb{R}^{4}\right)} \leq 2 c_{\kappa} \varepsilon^{\mu} r_{\varepsilon, \lambda, \gamma}^{2}, \quad\|\varphi\|_{\mathcal{C}^{4, \alpha}\left(S^{3}\right)} \leq \kappa r_{\varepsilon, \lambda, \gamma}^{2}, \quad\|\psi\|_{\mathcal{C}^{2, \alpha}\left(S^{3}\right)} \leq \kappa r_{\varepsilon, \lambda, \gamma}^{2}, \\
& \text { and }\left|\log \left(\tau / \tau_{*}\right)\right| \leq \kappa r_{\varepsilon, \lambda, \gamma}^{2} \log 1 / r_{\varepsilon, \lambda, \gamma}^{2} .
\end{aligned}
$$

Proof : The proof of these estimates follows from the result of Lemma 4.1 together with the assumption on the norms of $\varphi$ and $\psi$ and recall that a functions in $\mathcal{C}_{\mu}^{k, \alpha}\left(\mathbb{R}^{4}\right)$ are bounded by a constant times $\left(1+r^{2}\right)^{\mu / 2}$ and have their $\ell-t h$ partial derivatives that are bounded by $\left(1+r^{2}\right)^{(\mu-\ell) / 2}$, for $\ell=$ 
SINGULAR LIMITS FOR 4-DIMENSIONAL GENERAL STATIONARY

$1, \cdots, k+\alpha$

Indeed, let $c_{\kappa}$ denote constants which only depend on $\kappa$ (provided $\varepsilon, \lambda$ and $\gamma$ are chosen small enough), it follows from Lemma 4.1 and the estimates given by (42) and under the hypothesis (36), the coefficients of $r^{0}$ and $r^{1}$ vanish and hence, at least formally, the expansion of $H^{i}$ only involves powers of $r$ that are greater than or equal to 2 , then

$\left\|H^{i}\left(\varphi, \psi ; \cdot / R_{\varepsilon, \lambda, \gamma}\right)\right\|_{\mathcal{C}_{2}^{4, \alpha}\left(\bar{B}_{R_{\varepsilon, \lambda, \gamma}}\right)} \leq c_{\kappa} R_{\varepsilon, \lambda, \gamma}^{-2}\left(\|\varphi\|_{\mathcal{C}^{4, \alpha}\left(S^{3}\right)}+\|\psi\|_{\mathcal{C}^{2, \alpha}\left(S^{3}\right)}\right) \leq c_{\kappa} \varepsilon^{2}$.

* For $q \in[1,4)$.

Using the fact that $\|h\|_{\mathcal{C}_{\text {rad, }}^{4, \alpha}\left(\mathbb{R}^{4}\right)} \leq 2 c_{\kappa} \varepsilon^{\delta} r_{\varepsilon, \lambda, \gamma}^{2}$, then for all $x \in \bar{B}_{R_{\varepsilon, \lambda, \gamma}}$, $|h(x)| \leq c_{\kappa} r_{\varepsilon, \lambda, \gamma}^{2+\delta}$ tends to 0 as $\varepsilon, \lambda$ and $\gamma$ tend to 0 and from the asymptotic behavior of $H^{i}$ given by the estimate (45) then we get

$$
\left\|\left(1+|\cdot|^{2}\right)^{-4} e^{h}\left(e^{H^{i}\left(\varphi, \psi ; \cdot / R_{\varepsilon, \lambda, \gamma}\right)}-1\right)\right\|_{\mathcal{C}_{\mu-4}^{0, \alpha}\left(\bar{B}_{\left.R_{\varepsilon, \lambda, \gamma}\right)}\right.} \leq c_{\kappa} \varepsilon^{2}
$$

Again, using the fact that a function $w$ in $\mathcal{C}_{\mu}^{k, \alpha}\left(\mathbb{R}^{4}\right)$ are bounded by a constant times $\left(1+r^{2}\right)^{\mu / 2}$ and have their $\ell-t h$ partial derivatives that are bounded by $\left(1+r^{2}\right)^{(\mu-\ell) / 2}$, for $\ell=1, \cdots, k+\alpha\left(\right.$ a.e $\left|\nabla^{\ell} w\right| \leq c_{\kappa} r^{\mu-\ell}\|w\|_{\mathcal{C}_{\text {rad, }}^{4, \alpha}\left(\mathbb{R}^{4}\right)}$, $\left(1+r^{2}\right)^{(\mu-\ell) / 2} \sim r^{\mu-\ell}$ for $r$ very large) and provided $h \in \mathcal{C}_{\text {rad, }, \delta}^{4, \alpha}\left(\mathbb{R}^{4}\right)$ satisfy $\|h\|_{\mathcal{C}_{\text {rad }, \delta}^{4, \alpha}\left(\mathbb{R}^{4}\right)} \leq 2 c_{\kappa} \varepsilon^{\delta} r_{\varepsilon, \lambda, \gamma}^{2}$ and from the asymptotic behavior of $H^{i}$ given by the estimate $(45), \mu \in(1,2), q \in[1,4)$ and $\delta \in(0, \min (1,4-q))$, we deduce that

$$
\begin{aligned}
& \left\|\mid \lambda \varepsilon^{4-q}\left(\left|\nabla\left(u_{1}+h+H^{i}\left(\varphi, \psi ; \cdot / R_{\varepsilon, \lambda, \gamma}\right)\right)\right|^{q}-\left|\nabla\left(u_{1}+h\right)\right|^{q}\right)\right\|_{\mathcal{C}_{\mu-4}^{0, \alpha}\left(\bar{B}_{R_{\varepsilon, \lambda}, \gamma}\right)} \\
& \leq c_{\kappa} \lambda \varepsilon^{4-q} \sup _{r \leq R} r^{4-\mu}\left(\left|\nabla u_{1}\right|^{q-1}+|\nabla h|^{q-1}+\left|\nabla H^{i}\left(\varphi, \cdot / R_{\varepsilon, \lambda, \gamma}\right)\right|^{q-1}\right)\left|\nabla H^{i}\left(\varphi, \cdot / R_{\varepsilon, \lambda, \gamma}\right)\right| \\
& \leq c_{\kappa} \lambda \varepsilon^{6-q}\left[R_{\varepsilon, \lambda, \gamma}^{6-\mu-q}\right. \\
& \left.+R_{\varepsilon, \lambda, \gamma}^{6-\mu+\delta q-\delta-q}\|h\|_{\mathrm{C}_{\text {rad, }}^{4, \alpha}\left(\mathbb{R}^{4}\right)}^{q-1}+R_{\varepsilon, \lambda, \gamma}^{4-\mu+q}\left\|H^{i}\left(\varphi, \psi ; \cdot / R_{\varepsilon, \lambda, \gamma}\right)\right\|_{\mathcal{C}_{2}^{4, \alpha}\left(\bar{B}_{R_{\varepsilon, \lambda, \gamma}}\right)}^{q-1}\right] \\
& \leq c_{\kappa} \lambda \varepsilon^{6-q}\left[\varepsilon^{-6+\mu+q} r_{\varepsilon, \lambda, \gamma}^{6-\mu-q}+r_{\varepsilon, \lambda, \gamma}^{6-\mu+\delta q-\delta-q} \varepsilon^{-6+\mu-\delta q+\delta+q}\|h\|_{\mathcal{C}_{\text {rad, }}^{4, \alpha}\left(\mathbb{R}^{4}\right)}^{q-1}\right. \\
& \left.+\varepsilon^{-4+\mu-q} r_{\varepsilon, \lambda, \gamma}^{4-\mu+q}\left\|H^{i}\left(\varphi, \psi ; \cdot / R_{\varepsilon, \lambda, \gamma}\right)\right\|_{\mathcal{C}_{2}^{4, \alpha}\left(\bar{B}_{R_{\varepsilon, \lambda, \gamma}}\right)}^{q-1}\right] \\
& \leq c_{\kappa} \lambda\left[\varepsilon^{\mu} r_{\varepsilon, \lambda, \gamma}^{6-\mu-q}+r_{\varepsilon, \lambda, \gamma}^{6-\mu+\delta q-\delta-q} \varepsilon^{\mu+\delta(1-q)}\|h\|_{\mathcal{C}_{\text {rad, }}^{4, \alpha}}^{q-1}\left(\mathbb{R}^{4}\right)\right. \\
& \left.+r_{\varepsilon, \lambda, \gamma}^{4-\mu+q} \varepsilon^{\mu+2(1-q)}\left\|H^{i}\left(\varphi, \psi ; \cdot / R_{\varepsilon, \lambda, \gamma}\right)\right\|_{\mathcal{C}_{2}^{4, \alpha}\left(\bar{B}_{R_{\varepsilon, \lambda, \gamma}}\right)}^{q-1}\right] \\
& \leq c_{\kappa} \lambda\left(\varepsilon^{\mu} r_{\varepsilon, \lambda, \gamma}^{6-\mu-q}+\varepsilon^{\mu} r_{\varepsilon, \lambda, \gamma}^{4-\mu+\delta q-\delta+q}+\varepsilon^{\mu} r_{\varepsilon, \lambda, \gamma}^{4-\mu+q}\right) \\
& \leq c_{\kappa} \varepsilon^{\mu} r_{\varepsilon, \lambda, \gamma}^{2} \text {. }
\end{aligned}
$$


SINGULAR LIMITS FOR 4-DIMENSIONAL GENERAL STATIONARY

Q-KURAMOTO-SIVASHINSKY EQUATION (Q-KSE) WITH EXPONENTIAL

NONLINEARITY

Using the asymptotic behavior of $H^{i}$ given by the estimate (45) and $\mu \in(1,2)$, we get

$$
\left\|\mid \varepsilon^{2} \Delta\left(H^{i}\left(\varphi, \psi ; \cdot / R_{\varepsilon, \lambda, \gamma}\right)\right)\right\|_{\mathcal{C}_{\mu-4}^{0, \alpha}\left(\bar{B}_{R_{\varepsilon, \lambda, \gamma}}\right)} \leq c_{\kappa} \varepsilon^{\mu} r_{\varepsilon, \lambda, \gamma}^{4-\mu} \leq c_{\kappa} \varepsilon^{\mu} r_{\varepsilon, \lambda, \gamma}^{2}
$$

Making use of Proposition 3.1 together with (28) and the fact that $\mu \in$ $(1,2)$, we conclude that

$$
\|N(\varepsilon, \lambda, \gamma, \tau, \varphi, \psi ; 0)\|_{\mathcal{C}_{\mu}^{4, \alpha}\left(\mathbb{R}^{4}\right)} \leq c_{\kappa} \varepsilon^{\mu} r_{\varepsilon, \lambda, \gamma}^{2}
$$

To derive the second estimate, we use the fact that

$$
\begin{gathered}
\left\|\left(1+|\cdot|^{2}\right)^{-4} e^{H^{i}\left(\varphi, \psi ; \cdot / R_{\varepsilon, \lambda, \gamma}\right)+h}\left(e^{v_{2}}-e^{v_{1}}-v_{2}+v_{1}\right)\right\|_{\mathcal{C}_{\mu-4}^{0, \alpha}\left(\bar{B}_{R_{\varepsilon, \lambda, \gamma}}\right.} \leq \\
c_{\kappa} \varepsilon^{\mu} r_{\varepsilon, \lambda, \gamma}^{2}\left\|v_{2}-v_{1}\right\|_{\mathcal{C}_{\mu}^{4, \alpha}\left(\mathbb{R}^{4}\right)}
\end{gathered}
$$

and

$$
\begin{gathered}
\left\|\left(1+|\cdot|^{2}\right)^{-4} e^{h}\left(e^{H^{i}\left(\varphi, \psi ; \cdot / R_{\varepsilon, \lambda, \gamma}\right)}-1\right)\left(v_{2}-v_{1}\right)\right\|_{\mathcal{C}_{\mu-4}^{0, \alpha}\left(\bar{B}_{R_{\varepsilon}, \lambda, \gamma}\right)} \leq \\
c_{\kappa} \varepsilon^{2}\left\|v_{2}-v_{1}\right\|_{\mathcal{C}_{\mu}^{4, \alpha}\left(\mathbb{R}^{4}\right)},
\end{gathered}
$$

and

$$
\left\|\varepsilon^{2} \Delta\left(v_{2}-v_{1}\right)\right\|_{\mathcal{C}_{\mu-4}^{0, \alpha}\left(\bar{B}_{R_{\varepsilon, \lambda, \gamma}}\right)} \leq c_{\kappa} r_{\varepsilon, \lambda, \gamma}^{2}\left\|v_{2}-v_{1}\right\|_{\mathcal{C}_{\mu}^{4, \alpha}\left(\mathbb{R}^{4}\right)} .
$$

Provided $h \in \mathcal{C}_{r a d, \delta}^{4, \alpha}\left(\mathbb{R}^{4}\right)$ satisfy $\|h\|_{\mathcal{C}_{r a d, \delta}^{4, \alpha}\left(\mathbb{R}^{4}\right)} \leq 2 c_{\kappa} \varepsilon^{\delta} r_{\varepsilon, \lambda, \gamma}^{2}$, the fact that $|h(x)| \leq c_{\kappa} r_{\varepsilon, \lambda, \gamma}^{2+\delta}$ tends to 0 as $\varepsilon, \lambda$ and $\gamma$ tend to 0 , we deduce that

$$
\begin{aligned}
\left\|\left(1+|\cdot|^{2}\right)^{-4}\left(e^{h}-1\right)\left(v_{2}-v_{1}\right)\right\|_{\mathcal{C}_{\mu-4}^{0, \alpha}\left(\bar{B}_{R_{\varepsilon}, \lambda, \gamma}\right)} & \leq c_{\kappa}\|h\|_{\mathcal{C}_{r a d, \delta}^{4, \alpha}\left(\mathbb{R}^{4}\right)}\left\|v_{2}-v_{1}\right\|_{\mathcal{C}_{\mu}^{4, \alpha}\left(\mathbb{R}^{4}\right)} \\
& \leq c_{\kappa} \varepsilon^{\delta} r_{\varepsilon, \lambda, \gamma}^{2}\left\|v_{2}-v_{1}\right\|_{\mathcal{C}_{\mu}^{4, \alpha}\left(\mathbb{R}^{4}\right)} .
\end{aligned}
$$

Using the fact that a functions in $\mathcal{C}_{\mu}^{k, \alpha}\left(\mathbb{R}^{4}\right)$ are bounded by a constant times $\left(1+r^{2}\right)^{\mu / 2}$ and have their $\ell-t h$ partial derivatives that are bounded by $\left(1+r^{2}\right)^{(\mu-\ell) / 2}$, for $\ell=1, \cdots, k+\alpha$, (a.e $\left|\nabla^{\ell} w\right| \leq c_{\kappa} r^{\mu-\ell}\|w\|_{\mathcal{C}_{\mu}^{4, \alpha}\left(\mathbb{R}^{4}\right)},(1+$ $\left.r^{2}\right)^{(\mu-\ell) / 2} \sim r^{\mu-\ell}$ for $r$ very large) and provided $h \in \mathcal{C}_{r a d, \delta}^{4, \alpha}\left(\mathbb{R}^{4}\right)$ satisfy $\|h\|_{\mathcal{C}_{\text {rad, }}^{4, \alpha}\left(\mathbb{R}^{4}\right)} \leq 2 c_{\kappa} \varepsilon^{\delta} r_{\varepsilon, \lambda, \gamma}^{2}$ for $\delta \in(0, \min (1,4-q))$ and making use of Lemma 3.2 we deduce that 


$$
\begin{aligned}
& \| \mid \lambda \varepsilon^{4-q}\left(\mid \nabla\left(u_{1}+h+\left.H^{i}\left(\varphi, \psi ; \cdot / R_{\varepsilon, \lambda, \gamma}+v_{2}\right)\right|^{q}\right.\right. \\
& -\mid \nabla\left(u_{1}+h+\left.H^{i}\left(\varphi, \psi ; \cdot / R_{\varepsilon, \lambda, \gamma}+v_{1}\right)\right|^{q}\right) \|_{\mathcal{C}_{\mu-4}^{0, \alpha}\left(\bar{B}_{R_{\varepsilon, \lambda, \gamma}}\right)} \\
& \leq c_{\kappa} \lambda \varepsilon^{4-q} \sup _{r \leq R_{\varepsilon, \lambda, \gamma}} r^{4-\mu}\left[\left|\nabla\left(u_{1}+h+H^{i}\left(\varphi, \psi ; \cdot / R_{\varepsilon, \lambda, \gamma}\right)+v_{1}\right)\right|^{q-1}\right. \\
& \left.+\left|\nabla\left(v_{2}-v_{1}\right)\right|^{q-1}\right]\left|\nabla\left(v_{2}-v_{1}\right)\right| \\
& \leq c_{\kappa} \lambda \varepsilon^{4-q} \sup _{r \leq R_{\varepsilon, \lambda, \gamma}} r^{4-\mu}\left[\left|\nabla u_{1}\right|^{q-1}+|\nabla h|^{q-1}+\mid \nabla\left(\left.H^{i}\left(\varphi, \psi ; \cdot / R_{\varepsilon, \lambda, \gamma}\right)\right|^{q-1}\right.\right. \\
& \left.+\left|\nabla v_{1}\right|^{q-1}+\left|\nabla v_{2}\right|^{q-1}\right]\left|\nabla\left(v_{2}-v_{1}\right)\right| \\
& \leq c_{\kappa} \lambda \varepsilon^{4-q}\left[R_{\varepsilon, \lambda, \gamma}^{4-q}+R_{\varepsilon, \lambda, \gamma}^{4+\delta q-\delta-q}\|h\|_{\mathcal{C}_{\text {rad, } \delta}^{4, \alpha}}^{q-1}\left(\mathbb{R}^{4}\right)\right. \\
& +R_{\varepsilon, \lambda, \gamma}^{q+2}\left\|H^{i}\left(\varphi, \psi ; \cdot / R_{\varepsilon, \lambda, \gamma}\right)\right\|_{\mathcal{C}_{2}^{4, \alpha}\left(\bar{B}_{R_{\varepsilon, \lambda, \gamma}}\right)}^{q-1} \\
& \left.+R_{\varepsilon, \lambda, \gamma}^{4+\mu q-\mu-q} \sum_{i=1}^{2}\left\|v_{i}\right\|_{\mathcal{C}_{\mu}^{4, \alpha}\left(\mathbb{R}^{4}\right)}^{q-1}\right]\left\|v_{2}-v_{1}\right\|_{\mathcal{C}_{\mu}^{4, \alpha}\left(\mathbb{R}^{4}\right)} \\
& \leq c_{\kappa} \lambda\left(r_{\varepsilon, \lambda, \gamma}^{4-q}+r_{\varepsilon, \lambda, \gamma}^{4+\delta q-\delta-q} \varepsilon^{\delta(1-q)}\|h\|_{\mathcal{C}_{\text {rad, }}^{4, \alpha}\left(\mathbb{R}^{4}\right)}^{q-1}\right. \\
& +r_{\varepsilon, \lambda, \gamma}^{q+2} \varepsilon^{2(1-q)}\left\|H^{i}\left(\varphi, \psi ; \cdot / R_{\varepsilon, \lambda, \gamma}\right)\right\|_{\mathrm{C}_{2}^{4, \alpha}\left(\bar{B}_{R_{\varepsilon, \lambda, \gamma}}\right)}^{q-1} \\
& \left.+r_{\varepsilon, \lambda, \gamma}^{4+\mu q-\mu-q} \varepsilon^{\mu(1-q)} \sum_{i=1}^{2}\left\|v_{i}\right\|_{\mathcal{C}_{\mu}^{4, \alpha}\left(\mathbb{R}^{4}\right)}^{q-1}\right)\left\|v_{2}-v_{1}\right\|_{\mathcal{C}_{\mu}^{4, \alpha}\left(\mathbb{R}^{4}\right)} .
\end{aligned}
$$

Provided $v_{1}, v_{2} \in \mathcal{C}_{\mu}^{4, \alpha}\left(\mathbb{R}^{4}\right)$ satisfy $\left\|v_{i}\right\|_{\mathcal{C}_{\mu}^{4, \alpha}\left(\mathbb{R}^{4}\right)} \leq 2 c_{\kappa} \varepsilon^{\mu} r_{\varepsilon, \lambda, \gamma}^{2}$, the fact that $\|h\|_{\left.\mathcal{C}_{\text {rad, } \delta(\mathbb{R}}^{4, \alpha}\right)} \leq 2 c_{\kappa} \varepsilon^{\delta} r_{\varepsilon, \lambda, \gamma}^{2}$, the asymptotic behavior of $H^{i}$ given by the estimate (45) and for $\mu \in(1,2)$ and $q \in[1,4)$, using Proposition 3.1 and (28) we derive the desired estimate.

Reducing $\varepsilon_{\kappa}, \lambda_{\kappa}$ and $\gamma_{\kappa}$ if necessary, we can assume that,

$$
\bar{c}_{\kappa} r_{\varepsilon, \lambda, \gamma}^{2} \leq \frac{1}{2}
$$

for all $\varepsilon \in\left(0, \varepsilon_{\kappa}\right), \lambda \in\left(0, \lambda_{\kappa}\right)$ and $\gamma \in\left(0, \gamma_{\kappa}\right)$. Then, (43) and (44) in Lemma 5.1 are enough to show that

$$
v \longmapsto N(\varepsilon, \lambda, \gamma, \tau, \varphi, \psi ; v)
$$

is a contraction from

$$
\left\{v \in \mathcal{C}_{\mu}^{4, \alpha}\left(\mathbb{R}^{4}\right) \quad: \quad\|v\|_{\mathcal{C}_{\mu}^{4, \alpha}\left(\mathbb{R}^{4}\right)} \leq 2 c_{\kappa} \varepsilon^{\mu} r_{\varepsilon, \lambda, \gamma}^{2}\right\}
$$

into itself and hence has a unique fixed point $v(\varepsilon, \lambda, \gamma, \tau, \varphi, \psi ; \cdot)$ in this set. This fixed point is a solution of (41) in $B_{R_{\varepsilon, \lambda, \gamma}}$. 
* For $q=4$.

Recall that for $\|\hbar\|_{\mathcal{C}_{\text {rad, }}^{4, \alpha}\left(\mathbb{R}^{4}\right)} \leq 2 c_{\kappa} r_{\varepsilon, \lambda, \gamma}^{2}$ then for each $x \in \bar{B}_{R_{\varepsilon, \lambda, \gamma}}$

$$
|\hbar(x)| \leq c_{\kappa} r_{\varepsilon, \lambda, \gamma}^{2+\delta} \varepsilon^{-\delta} \leq\left\{\begin{array}{rll}
c_{\kappa} \varepsilon^{1+\delta / 2} & \text { for } & \varepsilon \geq \max (\lambda, \gamma) \\
c_{\kappa} \lambda^{1+\delta / 2} \varepsilon^{-\delta} & \text { for } & \lambda>\max (\varepsilon, \gamma) \\
c_{\kappa} \gamma^{1+\delta / 2} \varepsilon^{-\delta} & \text { for } & \gamma>\max (\varepsilon, \lambda)
\end{array}\right.
$$

which tends to 0 as $\varepsilon, \lambda$ and $\gamma$ tend to 0 , using the condition $\left(A_{\varepsilon, \lambda, \gamma}\right)$. Then

$$
\left\|\left(1+|\cdot|^{2}\right)^{-4} e^{\hbar}\left(e^{H^{i}\left(\varphi, \psi ; \cdot / R_{\varepsilon, \lambda, \gamma}\right)}-1\right)\right\|_{\mathcal{C}_{\mu-4}^{0, \alpha}\left(\bar{B}_{\left.R_{\varepsilon, \lambda, \gamma}\right)}\right.} \leq c_{\kappa} \varepsilon^{2},
$$

Again, using the fact that a functions $w$ in $\mathcal{C}_{\mu}^{k, \alpha}\left(\mathbb{R}^{4}\right)$ are bounded by a constant times $\left(1+r^{2}\right)^{\mu / 2}$ and have their $\ell-t h$ partial derivatives that are bounded by $\left(1+r^{2}\right)^{(\mu-\ell) / 2}$, for $\ell=1, \cdots, k+\alpha\left(\right.$ a.e $\left|\nabla^{\ell} w\right| \leq c_{\kappa} r^{\mu-\ell}\|w\|_{\mathcal{C}_{\text {rad, }}^{4, \alpha}\left(\mathbb{R}^{4}\right)}$, $\left(1+r^{2}\right)^{(\mu-\ell) / 2} \sim r^{\mu-\ell}$ for $r$ very large) and provided $\hbar \in \mathcal{C}_{\text {rad, }, \delta}^{4, \alpha}\left(\mathbb{R}^{4}\right)$ satisfy $\|\hbar\|_{\mathcal{C}_{\text {rad, }}^{4, \alpha}\left(\mathbb{R}^{4}\right)} \leq 2 c_{\kappa} r_{\varepsilon, \lambda, \gamma}^{2}$ and from the asymptotic behavior of $H^{i}$ given by the estimate $(45), \mu \in(1,2), q=4, \delta \in(0,1)$ and using condition $\left(A_{\varepsilon, \lambda, \gamma}\right)$, we deduce that

$$
\begin{aligned}
& \left\|\mid \lambda\left(\left|\nabla\left(u_{1}+\hbar+H^{i}\left(\varphi, \psi ; \cdot / R_{\varepsilon, \lambda, \gamma}\right)\right)\right|^{4}-\left|\nabla\left(u_{1}+\hbar\right)\right|^{4}\right)\right\|_{\mathcal{C}_{\mu-4}^{0, \alpha}\left(\bar{B}_{R_{\varepsilon, \lambda, \gamma}}\right)} \\
& \leq c_{\kappa} \lambda \sup _{r \leq R_{\varepsilon, \lambda, \gamma}} r^{4-\mu}\left(\left|\nabla u_{1}\right|^{3}+|\nabla \hbar|^{3}+\left|\nabla H^{i}\left(\varphi, \cdot / R_{\varepsilon, \lambda, \gamma}\right)\right|^{3}\right)\left|\nabla H^{i}\left(\varphi, \cdot / R_{\varepsilon, \lambda, \gamma}\right)\right| \\
& \leq c_{\kappa} \lambda \varepsilon^{2}\left[R_{\varepsilon, \lambda, \gamma}^{2-\mu}+R_{\varepsilon, \lambda, \gamma}^{2-\mu+3 \delta}\|\hbar\|_{\mathcal{C}_{r a d, \delta}^{4, \alpha}\left(\mathbb{R}^{4}\right)}^{3}+R_{\varepsilon, \lambda, \gamma}^{8-\mu}\left\|H^{i}\left(\varphi, \psi ; \cdot / R_{\varepsilon, \lambda, \gamma}\right)\right\|_{\mathcal{C}_{2}^{4, \alpha}\left(\bar{B}_{R_{\varepsilon, \lambda, \gamma}}\right)}^{3}\right] \\
& \leq c_{\kappa} \lambda \varepsilon^{2}\left[\varepsilon^{-2+\mu} r_{\varepsilon, \lambda, \gamma}^{2-\mu}+r_{\varepsilon, \lambda, \gamma}^{2-\mu+3 \delta} \varepsilon^{-2+\mu-3 \delta}\|\hbar\|_{\mathcal{C}_{r, \alpha}^{4, \alpha, \delta}}^{3}\left(\mathbb{R}^{4}\right)\right. \\
& \left.+\varepsilon^{-8+\mu} r_{\varepsilon, \lambda, \gamma}^{8-\mu}\left\|H^{i}\left(\varphi, \psi ; \cdot / R_{\varepsilon, \lambda, \gamma}\right)\right\|_{\mathcal{C}_{2}^{4, \alpha}\left(\bar{B}_{R_{\varepsilon, \lambda, \gamma}}\right)}^{3}\right] \\
& \leq c_{\kappa} \lambda\left[\varepsilon^{\mu} r_{\varepsilon, \lambda, \gamma}^{2-\mu}+r_{\varepsilon, \lambda, \gamma}^{2-\mu+3 \delta} \varepsilon^{\mu-3 \delta}\|\hbar\|_{\mathcal{C}_{r a d, \delta}^{4, \alpha}\left(\mathbb{R}^{4}\right)}^{3}\right. \\
& \left.\quad+r_{\varepsilon, \lambda, \gamma}^{8-\mu} \varepsilon^{\mu-6}\left\|H^{i}\left(\varphi, \psi ; \cdot / R_{\varepsilon, \lambda, \gamma}\right)\right\|_{\mathcal{C}_{2}^{4, \alpha}\left(\bar{B}_{R_{\varepsilon, \lambda, \gamma}}\right)}^{3}\right] \\
& \leq c_{\kappa} \lambda\left(\varepsilon^{\mu} r_{\varepsilon, \lambda, \gamma}^{2-\mu}+\varepsilon^{\mu} r_{\varepsilon, \lambda, \gamma}^{2-\mu}\left(r_{\varepsilon, \lambda, \gamma}^{2+\delta} \varepsilon^{-\delta}\right)^{3}+\varepsilon^{\mu} r_{\varepsilon, \lambda, \gamma}^{8-\mu}\right) \\
& \leq c_{\kappa} \varepsilon^{\mu} r_{\varepsilon, \lambda, \gamma}^{2}\left(r_{\varepsilon, \lambda, \gamma}^{2-\mu}+r_{\varepsilon, \lambda, \gamma}^{2-\mu}\left(r_{\varepsilon, \lambda, \gamma}^{2+\delta} \varepsilon^{-\delta}\right)^{3}+r_{\varepsilon, \lambda, \gamma}^{8-\mu}\right) \\
& \quad \leq c_{\kappa} \varepsilon^{\mu} r_{\varepsilon, \lambda, \gamma}^{2}
\end{aligned}
$$

Again, using the asymptotic behavior of $H^{i}$ given by the estimate (45) and $\mu \in(1,2)$, we get

$$
\left\|\varepsilon^{2} \Delta\left(H^{i}\left(\varphi, \psi ; \cdot / R_{\varepsilon, \lambda, \gamma}\right)\right)\right\|_{\mathcal{C}_{\mu-4}^{0, \alpha}\left(\bar{B}_{R_{\varepsilon, \lambda, \gamma}}\right)} \leq c_{\kappa} \varepsilon^{\mu} r_{\varepsilon, \lambda, \gamma}^{4-\mu} \leq c_{\kappa} \varepsilon^{\mu} r_{\varepsilon, \lambda, \gamma}^{2}
$$


SINGULAR LIMITS FOR 4-DIMENSIONAL GENERAL STATIONARY

Q-KURAMOTO-SIVASHINSKY EQUATION (Q-KSE) WITH EXPONENTIAL

Making use of Proposition 3.1 together with (28), we conclude that

$$
\|N(\varepsilon, \lambda, \gamma, \tau, \varphi, \psi ; 0)\|_{\mathcal{C}_{\mu}^{4, \alpha}\left(\mathbb{R}^{4}\right)} \leq c_{\kappa} \varepsilon^{\mu} r_{\varepsilon, \lambda, \gamma}^{2} .
$$

To derive the second estimate, we use the fact that $|\hbar(x)| \longrightarrow 0$ as $\varepsilon, \lambda$ and $\gamma$ tend to 0 under the condition $\left(A_{\varepsilon, \lambda, \gamma}\right)$ and then

$$
\begin{gathered}
\left\|\left(1+|\cdot|^{2}\right)^{-4} e^{H^{i}\left(\varphi, \psi ; \cdot / R_{\varepsilon, \lambda, \gamma}\right)+\hbar}\left(e^{v_{2}}-e^{v_{1}}-v_{2}+v_{1}\right)\right\|_{\mathcal{C}_{\mu-4}^{0, \alpha}\left(\bar{B}_{R_{\varepsilon, \lambda, \gamma}}\right)} \\
\leq c_{\kappa} \varepsilon^{\mu} r_{\varepsilon, \lambda, \gamma}^{2}\left\|v_{2}-v_{1}\right\|_{\mathcal{C}_{\mu}^{4, \alpha}\left(\mathbb{R}^{4}\right)}, \\
\left\|\left(1+|\cdot|^{2}\right)^{-4} e^{\hbar}\left(e^{H^{i}\left(\varphi, \psi ; \cdot / R_{\varepsilon, \lambda, \gamma}\right)}-1\right)\left(v_{2}-v_{1}\right)\right\|_{\mathcal{C}_{\mu-4}^{0, \alpha}\left(\bar{B}_{R_{\varepsilon, \lambda, \gamma}}\right)} \\
\leq c_{\kappa} \varepsilon^{2}\left\|v_{2}-v_{1}\right\|_{\mathcal{C}_{\mu}^{4, \alpha}\left(\mathbb{R}^{4}\right)},
\end{gathered}
$$

and

$$
\left\|\varepsilon^{2} \Delta\left(v_{2}-v_{1}\right)\right\|_{\mathcal{C}_{\mu-4}^{0, \alpha}\left(\bar{B}_{\left.R_{\varepsilon, \lambda, \gamma}\right)}\right.} \leq c_{\kappa} r_{\varepsilon, \lambda, \gamma}^{2}\left\|v_{2}-v_{1}\right\|_{\mathcal{C}_{\mu}^{4, \alpha}\left(\mathbb{R}^{4}\right)} .
$$

Provided $h \in \mathcal{C}_{\text {rad, }}^{4, \alpha}\left(\mathbb{R}^{4}\right)$ satisfy $\|\hbar\|_{\mathcal{C}_{\text {rad, }}^{4, \alpha}\left(\mathbb{R}^{4}\right)} \leq 2 c_{\kappa} r_{\varepsilon, \lambda, \gamma}^{2}$ and $|\hbar(x)| \longrightarrow 0$ as $\varepsilon, \lambda$ and $\gamma$ tend to 0 , under the condition $\left(A_{\varepsilon, \lambda, \gamma}\right)$, we deduce that

$$
\begin{aligned}
\left\|\left(1+|\cdot|^{2}\right)^{-4}\left(e^{\hbar}-1\right)\left(v_{2}-v_{1}\right)\right\|_{\mathcal{C}_{\mu-4}^{0, \alpha}\left(\bar{B}_{R_{\varepsilon, \lambda, \gamma}}\right)} & \leq c_{\kappa}\|\hbar\|_{\mathcal{C}_{\text {rad, }}^{4, \alpha}\left(\mathbb{R}^{4}\right)}\left\|v_{2}-v_{1}\right\|_{\mathcal{C}_{\mu}^{4, \alpha}\left(\mathbb{R}^{4}\right)} \\
& \leq c_{\kappa} r_{\varepsilon, \lambda, \gamma}^{2}\left\|v_{2}-v_{1}\right\|_{\mathcal{C}_{\mu}^{4, \alpha}\left(\mathbb{R}^{4}\right)} .
\end{aligned}
$$

Using the fact that a functions in $\mathcal{C}_{\mu}^{k, \alpha}\left(\mathbb{R}^{4}\right)$ are bounded by a constant times $\left(1+r^{2}\right)^{\mu / 2}$ and have their $\ell-t h$ partial derivatives that are bounded by $\left(1+r^{2}\right)^{(\mu-\ell) / 2}$, for $\ell=1, \cdots, k+\alpha$, (a.e $\left|\nabla^{\ell} w\right| \leq c_{\kappa} r^{\mu-\ell}\|w\|_{\mathcal{C}_{\mu}^{4, \alpha}\left(\mathbb{R}^{4}\right)}$, $\left(1+r^{2}\right)^{(\mu-\ell) / 2} \sim r^{\mu-\ell}$ for $r$ very large) and provided $\hbar \in \mathcal{C}_{\text {rad, }}^{4, \alpha}\left(\mathbb{R}^{4}\right)$ satisfy $\|\hbar\|_{\mathcal{C}_{\text {rad, }}^{4, \alpha}\left(\mathbb{R}^{4}\right)} \leq 2 c_{\kappa} r_{\varepsilon, \lambda, \gamma}^{2}$ for $\delta \in(0,1)$ and making use of Lemma 3.2, we deduce that 


$$
\begin{aligned}
& \| \mid \lambda\left(\mid \nabla\left(u_{1}+h+\left.H^{i}\left(\varphi, \psi ; \cdot / R_{\varepsilon, \lambda, \gamma}+v_{2}\right)\right|^{4}\right.\right. \\
& -\mid \nabla\left(u_{1}+h+\left.H^{i}\left(\varphi, \psi ; \cdot / R_{\varepsilon, \lambda, \gamma}+v_{1}\right)\right|^{4}\right) \|_{\mathcal{C}_{\mu-4}^{0, \alpha}\left(\bar{B}_{R_{\varepsilon}, \lambda, \gamma}\right)} \\
& \leq c_{\kappa} \lambda \sup _{r \leq R_{\varepsilon, \lambda, \gamma}} r^{4-\mu}\left[\left|\nabla\left(u_{1}+h+H^{i}\left(\varphi, \psi ; \cdot / R_{\varepsilon, \lambda, \gamma}\right)+v_{1}\right)\right|^{3}+\left|\nabla\left(v_{2}-v_{1}\right)\right|^{3}\right]\left|\nabla\left(v_{2}-v_{1}\right)\right| \\
& \leq c_{\kappa} \lambda \sup _{r \leq R_{\varepsilon, \lambda, \gamma}} r^{4-\mu}\left[\left|\nabla u_{1}\right|^{3}+|\nabla h|^{3}+\mid \nabla\left(\left.H^{i}\left(\varphi, \psi ; \cdot / R_{\varepsilon, \lambda, \gamma}\right)\right|^{3}\right.\right. \\
& \left.+\left|\nabla v_{1}\right|^{q-1}+\left|\nabla v_{2}\right|^{3}\right]\left|\nabla\left(v_{2}-v_{1}\right)\right| \\
& \leq c_{\kappa} \lambda\left[1+R_{\varepsilon, \lambda, \gamma}^{3 \delta}\|h\|_{\mathcal{C}_{r a d, \delta}^{4, \alpha}\left(\mathbb{R}^{4}\right)}^{3}+R_{\varepsilon, \lambda, \gamma}^{6}\left\|H^{i}\left(\varphi, \psi ; \cdot / R_{\varepsilon, \lambda, \gamma}\right)\right\|_{\mathcal{C}_{2}^{4, \alpha}\left(\bar{B}_{R_{\varepsilon}, \lambda, \gamma}\right)}^{3}\right. \\
& \left.+R_{\varepsilon, \lambda, \gamma}^{3 \mu} \sum_{i=1}^{2}\left\|v_{i}\right\|_{\mathcal{C}_{\mu}^{4, \alpha}\left(\mathbb{R}^{4}\right)}^{3}\right]\left\|v_{2}-v_{1}\right\|_{\mathcal{C}_{\mu}^{4, \alpha}\left(\mathbb{R}^{4}\right)} \\
& \leq c_{\kappa} \lambda\left(1+r_{\varepsilon, \lambda, \gamma}^{3 \delta} \varepsilon^{-3 \delta}\|h\|_{\mathcal{C}_{r a d, \delta}^{4, \alpha}\left(\mathbb{R}^{4}\right)}^{3}+r_{\varepsilon, \lambda, \gamma}^{6} \varepsilon^{-6}\left\|H^{i}\left(\varphi, \psi ; \cdot / R_{\varepsilon, \lambda, \gamma}\right)\right\|_{\mathcal{C}_{2}^{4, \alpha}\left(\bar{B}_{R_{\varepsilon, \lambda, \gamma}}\right)}^{3}\right. \\
& \left.+r_{\varepsilon, \lambda, \gamma}^{3 \mu} \varepsilon^{-3 \mu} \sum_{i=1}^{2}\left\|v_{i}\right\|_{\mathcal{C}_{\mu}^{4, \alpha}\left(\mathbb{R}^{4}\right)}^{3}\right)\left\|v_{2}-v_{1}\right\|_{\mathcal{C}_{\mu}^{4, \alpha}\left(\mathbb{R}^{4}\right)} \cdot
\end{aligned}
$$

Provided $v_{1}, v_{2} \in \mathcal{C}_{\mu}^{4, \alpha}\left(\mathbb{R}^{4}\right)$ satisfy $\left\|v_{i}\right\|_{\mathcal{C}_{\mu}^{4, \alpha}\left(\mathbb{R}^{4}\right)} \leq 2 c_{\kappa} \varepsilon^{\mu} r_{\varepsilon, \lambda, \gamma}^{2}$, the fact that $\|h\|_{\mathcal{C}_{\text {rad, }}^{4, \alpha}\left(\mathbb{R}^{4}\right)} \leq 2 c_{\kappa} r_{\varepsilon, \lambda, \gamma}^{2}$ and $|\hbar(x)| \leq c_{\kappa} r_{\varepsilon, \lambda, \gamma}^{2+\delta} \varepsilon^{-\delta} \longrightarrow 0$ as $\varepsilon, \lambda$ and $\gamma$ tend to 0 under the condition $\left(A_{\varepsilon, \lambda, \gamma}\right)$, the asymptotic behavior of $H^{i}$ given by the estimate (45) and for $\mu \in(1,2)$ and $q=4$, using Proposition 3.1 and (28) we derive the desired estimate.

Reducing $\varepsilon_{\kappa}, \lambda_{\kappa}$ and $\gamma_{\kappa}$ if necessary, we can assume that,

$$
\bar{c}_{\kappa} r_{\varepsilon, \lambda, \gamma}^{2} \leq \frac{1}{2}
$$

for all $\varepsilon \in\left(0, \varepsilon_{\kappa}\right), \lambda \in\left(0, \lambda_{\kappa}\right)$ and $\gamma \in\left(0, \gamma_{\kappa}\right)$ satisfying $\left(A_{\varepsilon, \lambda, \gamma}\right)$. Then, (43) and (44) in Lemma 5.1 are enough to show that

$$
v \longmapsto N(\varepsilon, \lambda, \gamma, \tau, \varphi, \psi ; v)
$$

is a contraction from

$$
\left\{v \in \mathcal{C}_{\mu}^{4, \alpha}\left(\mathbb{R}^{4}\right) \quad: \quad\|v\|_{\mathcal{C}_{\mu}^{4, \alpha}\left(\mathbb{R}^{4}\right)} \leq 2 c_{\kappa} \varepsilon^{\mu} r_{\varepsilon, \lambda, \gamma}^{2}\right\}
$$

into itself and hence has a unique fixed point $v(\varepsilon, \lambda, \gamma, \tau, \varphi, \psi ; \cdot)$ in this set. This fixed point is a solution of (41) in $B_{R_{\varepsilon, \lambda, \gamma}}$.

We summarize this in the : 
SINGULAR LIMITS FOR 4-DIMENSIONAL GENERAL STATIONARY

Q-KURAMOTO-SIVASHINSKY EQUATION (Q-KSE) WITH EXPONENTIAL

NONLINEARITY

Proposition 5.1. Given $\kappa>1$, there exist $c_{\kappa}>0$ (only depending on $\kappa$ ) such that given $\varphi \in \mathrm{C}^{4, \alpha}\left(S^{3}\right), \psi \in \mathfrak{C}^{2, \alpha}\left(S^{3}\right)$ satisfying (36) and $\tau>0$ satisfying

$\left|\log \left(\tau / \tau_{*}\right)\right| \leq \kappa r_{\varepsilon, \lambda, \gamma}^{2} \log 1 / r_{\varepsilon, \lambda, \gamma}^{2}, \quad\|\varphi\|_{\mathcal{e}^{4, \alpha}\left(S^{3}\right)} \leq \kappa r_{\varepsilon, \lambda, \gamma}^{2}$ and $\|\psi\|_{\mathrm{e}^{2, \alpha}\left(S^{3}\right)} \leq \kappa r_{\varepsilon, \lambda, \gamma}^{2}$, then

i) for $q \in[1,4)$, there exist $\varepsilon_{\kappa}>0, \lambda_{\kappa}>0, \gamma_{\kappa}>0$ and $c_{\kappa}>0$ (only depending on $\kappa)$ such that for all $\varepsilon \in\left(0, \varepsilon_{\kappa}\right), \lambda \in\left(0, \lambda_{\kappa}\right)$ and $\gamma \in\left(0, \gamma_{\kappa}\right)$, the function

$$
u(\varepsilon, \lambda, \gamma, \tau, \varphi, \psi ; \cdot):=u_{1}+h+H^{i}\left(\varphi, \psi ; \cdot / R_{\varepsilon, \lambda, \gamma}\right)+v(\varepsilon, \lambda, \gamma, \tau, \varphi, \psi ; \cdot),
$$

solves (39) in $\bar{B}_{R_{\varepsilon, \lambda, \gamma}}$. In addition

$$
\|v(\varepsilon, \lambda, \gamma, \tau, \varphi, \psi ; \cdot)\|_{\mathcal{C}_{\mu}^{4, \alpha}\left(\mathbb{R}^{4}\right)} \leq 2 c_{\kappa} \varepsilon^{\mu} r_{\varepsilon, \lambda, \gamma}^{2}
$$

and

$$
\|h\|_{\mathcal{C}_{\text {rad, }, \alpha}^{4,}\left(\mathbb{R}^{4}\right)} \leq 2 c_{\kappa} \varepsilon^{\delta} r_{\varepsilon, \lambda, \gamma}^{2}
$$

ii) for $q=4$, there exist $\varepsilon_{\kappa}>0, \lambda_{\kappa}>0, \gamma_{\kappa}>0$ and $c_{\kappa}>0$ such that for all $\varepsilon \in\left(0, \varepsilon_{\kappa}\right), \lambda \in\left(0, \lambda_{\kappa}\right)$ and $\gamma \in\left(0, \gamma_{\kappa}\right)$ satisfying $\left(A_{\varepsilon, \lambda, \gamma}\right)$, the function

$$
u(\varepsilon, \lambda, \gamma, \tau, \varphi, \psi ; \cdot):=u_{1}+\hbar+H^{i}\left(\varphi, \psi ; \cdot / R_{\varepsilon, \lambda, \gamma}\right)+v(\varepsilon, \lambda, \gamma, \tau, \varphi, \psi ; \cdot),
$$

solves (39) in $\bar{B}_{R_{\varepsilon, \lambda, \gamma}}$. In addition

$$
\|v(\varepsilon, \lambda, \gamma, \tau, \varphi, \psi ; \cdot)\|_{\mathcal{C}_{\mu}^{4, \alpha}\left(\mathbb{R}^{4}\right)} \leq 2 c_{\kappa} \varepsilon^{\mu} r_{\varepsilon, \lambda, \gamma}^{2}
$$

and

$$
\|\hbar\|_{\mathcal{C}_{\text {rad, }}^{4, \alpha}\left(\mathbb{R}^{4}\right)} \leq 2 c_{\kappa} r_{\varepsilon, \lambda, \gamma}^{2}
$$

Observe that the function $v(\varepsilon, \lambda, \gamma, \tau, \varphi, \psi ; \cdot)$ being obtained as a fixed point for contraction mapping, it depends continuously on the parameters $\tau$.

\section{The second nonlinear Dirichlet problem}

For all $(\varepsilon, \lambda, \gamma) \in\left(0, r_{0}^{2}\right)^{3}$, we recall that

$$
r_{\varepsilon, \lambda, \gamma}:=\max (\sqrt{\varepsilon}, \sqrt{\lambda}, \sqrt{\gamma})
$$

Recall that $G(x, \cdot)$ denotes the unique solution of

$$
\Delta^{2} G(x, \cdot)=64 \pi^{2} \delta_{x}
$$


SINGULAR LIMITS FOR 4-DIMENSIONAL GENERAL STATIONARY

Q-KURAMOTO-SIVASHINSKY EQUATION (Q-KSE) WITH EXPONENTIAL

in $\Omega$, with $G(x, \cdot)=\Delta G(x, \cdot)=0$ on $\partial \Omega$. In addition, the following decomposition holds

$$
G(x, y)=-8 \log |x-y|+R(x, y)
$$

where $y \longmapsto R(x, y)$ is a smooth function.

Lemma 6.1. There exists $C>0$ such that for all $x, y \in \Omega, x \neq y$, we have that

$$
\left|\nabla^{i} G(x, y)\right| \leq C|x-y|^{-i}, i \geq 1 .
$$

Proof. This estimate is originally due to Krasovski $i$ [12] and some reference therein.

Given $x^{1}, \ldots, x^{m} \in \Omega$. The data we will need are the following :

(i) Points $Y:=\left(y^{1}, \ldots, y^{m}\right) \in \Omega^{m}$ close enough to $X:=\left(x^{1}, \ldots, x^{m}\right)$.

(ii) Parameters $\tilde{\eta}:=\left(\tilde{\eta}^{1}, \ldots, \tilde{\eta}^{m}\right) \in \mathbb{R}^{m}$ close to 0 .

(iii) Boundary data $\Phi:=\left(\varphi^{1}, \ldots, \varphi^{m}\right) \in\left(\mathcal{C}^{4, \alpha}\left(S^{3}\right)\right)^{m}$ and $\Psi:=\left(\psi^{1}, \ldots, \psi^{m}\right) \in$ $\left(\mathfrak{C}^{2, \alpha}\left(S^{3}\right)\right)^{m}$ each of which satisfies (38).

With all these data, we define

$$
\tilde{\mathbf{u}}:=\sum_{j=1}^{m}\left(1+\tilde{\eta}^{j}\right) G\left(y^{j}, \cdot\right)+\sum_{j=1}^{m} \chi_{r_{0}}\left(\cdot-y^{j}\right) H^{e}\left(\varphi^{j}, \psi^{j} ;\left(\cdot-y^{j}\right) / r_{\varepsilon, \lambda, \gamma}\right)
$$

where $\chi_{r_{0}}$ is a cutoff function identically equal to 1 in $B_{r_{0} / 2}$ and identically equal to 0 outside $B_{r_{0}}$.

Recall that we have defined $\rho>0$ by

$$
\rho^{4}=\frac{384 \varepsilon^{4}}{\left(1+\varepsilon^{2}\right)^{4}}
$$

We fix

$$
q \in[1,4] .
$$

We would like to find a solution of the equation

$$
\Delta^{2} u-\gamma \Delta u-\lambda|\nabla u|^{q}-\rho^{4} e^{u}=0,
$$

which is defined in $\bar{\Omega}_{r_{\varepsilon, \lambda, \gamma}}(Y)$ and which is a perturbation of $\tilde{\mathbf{u}}$. Writing $u=\tilde{\mathbf{u}}+\tilde{v}$, this amounts to solve

$$
\Delta^{2} \tilde{v}=\rho^{4} e^{\tilde{\mathbf{u}}+\tilde{v}}-\Delta^{2} \tilde{\mathbf{u}}+\gamma \Delta(\tilde{\mathbf{u}}+\tilde{v})+\lambda|\nabla(\tilde{\mathbf{u}}+\tilde{v})|^{q} .
$$

We need to define an auxiliary weighted space : 
SINGULAR LIMITS FOR 4-DIMENSIONAL GENERAL STATIONARY

Q-KURAMOTO-SIVASHINSKY EQUATION (Q-KSE) WITH EXPONENTIAL

NONLINEARITY

Definition 6.1. Given $\bar{r} \in\left(0, r_{0} / 2\right), k \in \mathbb{R}, \alpha \in(0,1)$ and $\nu \in \mathbb{R}$, we define the Hölder weighted space $\mathcal{C}_{\nu}^{k, \alpha}\left(\bar{\Omega}_{\bar{r}}(X)\right)$ as the space of functions $w \in$ $\mathrm{C}^{k, \alpha}\left(\bar{\Omega}_{\bar{r}}(X)\right)$ which is endowed with the norm

$\|w\|_{\mathrm{e}_{\nu}^{k, \alpha}\left(\bar{\Omega}_{\bar{r}}(X)\right)}:=\|w\|_{\mathrm{e}^{k, \alpha}\left(\bar{\Omega}_{r_{0} / 2}(X)\right)}+\sum_{j=1}^{m} \sup _{r \in\left[\bar{r}, r_{0} / 2\right)}\left(r^{-\nu}\left\|w\left(x^{j}+r \cdot\right)\right\|_{\mathrm{e}^{k, \alpha}\left(\bar{B}_{2}-B_{1}\right)}\right)$.

For all $\sigma \in\left(0, r_{0} / 2\right)$ and all $Y \in \Omega^{m}$ such that $\|X-Y\| \leq r_{0} / 2$, we denote by

$$
\tilde{\varepsilon}_{\sigma, Y}: \mathcal{C}_{\nu}^{0, \alpha}\left(\bar{\Omega}_{\sigma}(Y)\right) \longrightarrow \mathcal{C}_{\nu}^{0, \alpha}\left(\bar{\Omega}^{*}(Y)\right),
$$

the extension operator defined by $\tilde{\mathcal{E}}_{\sigma, Y}(f)=f$ in $\bar{\Omega}_{\sigma}(Y)$

$$
\tilde{\mathcal{E}}_{\sigma, Y}(f)\left(y^{i}+x\right)=\tilde{\chi}\left(\frac{|x|}{\sigma}\right) f\left(y^{i}+\sigma \frac{x}{|x|}\right)
$$

for each $j=1, \ldots, m$ and $\tilde{\mathcal{E}}_{\sigma, Y}(f)=0$ in each $B_{\sigma / 2}\left(y^{j}\right)$, where $t \longmapsto \tilde{\chi}(t)$ is a cutoff function identically equal to 1 for $t \geq 1$ and identically equal to 0 for $t \leq 1 / 2$. It is easy to check that there exists a constant $c=c(\nu)>0$ only depending on $\nu$ such that

$$
\left\|\tilde{\mathcal{E}}_{\sigma, Y}(w)\right\|_{\mathcal{C}_{\nu}^{0, \alpha}\left(\bar{\Omega}^{*}(X)\right)} \leq c\|w\|_{\mathcal{C}_{\nu}^{0, \alpha}\left(\bar{\Omega}_{\sigma}(X)\right)} .
$$

We fix

$$
\nu \in(-1,0)
$$

and denote by $\tilde{\mathcal{G}}_{\nu, Y}$ the right inverse provided by Proposition 4.1. Clearly, it is enough to find $\tilde{v} \in \mathcal{C}_{\nu}^{4, \alpha}\left(\Omega^{*}(Y)\right)$ solution of

$$
\tilde{v}=\tilde{N}(\varepsilon, \lambda, \gamma, \tilde{\eta}, Y, \Phi, \Psi ; \tilde{v})
$$

where we have defined

$$
\begin{gathered}
\tilde{N}(\tilde{v}):=\tilde{N}(\varepsilon, \lambda, \gamma, \tilde{\eta}, Y, \Phi, \Psi ; \tilde{v}) \\
:=\tilde{\mathcal{G}}_{\nu, Y} \circ \tilde{\mathcal{E}}_{r_{\varepsilon, \lambda, \gamma}, Y}\left(\rho^{4} e^{\tilde{\mathbf{u}}+\tilde{v}}-\Delta^{2} \tilde{\mathbf{u}}+\gamma \Delta(\tilde{\mathbf{u}}+\tilde{v})+\lambda|\nabla(\tilde{\mathbf{u}}+\tilde{v})|^{q}\right) . \\
:=\tilde{\mathcal{G}}_{\nu, Y} \circ \tilde{\mathcal{E}}_{r_{\varepsilon, \lambda, \gamma}, Y}(\tilde{S}(v))
\end{gathered}
$$

Given $\kappa>0$ (whose value will be fixed later on), we further assume that $\Phi$ and $\Psi$ satisfy

$$
\|\Phi\|_{\left(\mathcal{C}^{4, \alpha}\left(S^{3}\right)\right)^{m}} \leq \kappa r_{\varepsilon, \lambda, \gamma}^{2}, \quad \text { and } \quad\|\Psi\|_{\left(\mathcal{C}^{2, \alpha}\left(S^{3}\right)\right)^{m}} \leq \kappa r_{\varepsilon, \lambda, \gamma}^{2} .
$$


SINGULAR LIMITS FOR 4-DIMENSIONAL GENERAL STATIONARY

Q-KURAMOTO-SIVASHINSKY EQUATION (Q-KSE) WITH EXPONENTIAL

NONLINEARITY

Moreover, we assume that the parameters $\tilde{\eta}$ and the points $Y$ are chosen to satisfy

$$
|\tilde{\eta}| \leq \kappa r_{\varepsilon, \lambda, \gamma}^{2}, \quad \text { and } \quad\|Y-X\| \leq \kappa r_{\varepsilon, \lambda, \gamma} .
$$

Then, the following result holds :

Lemma 6.2. Given $\kappa>1$. There exist $\varepsilon_{\kappa}>0, \lambda_{\kappa}>0, \gamma_{\kappa}>0, c_{\kappa}>0$ and $\bar{c}_{\kappa}>0$ such that, for all $\varepsilon \in\left(0, \varepsilon_{\kappa}\right), \lambda \in\left(0, \lambda_{\kappa}\right)$ and $\gamma \in\left(0, \gamma_{\kappa}\right)$, we have

$$
\|\tilde{N}(\varepsilon, \lambda, \gamma, \tilde{\eta}, Y, \Phi, \Psi ; 0)\|_{\mathcal{C}_{\nu}^{4, \alpha}\left(\bar{\Omega}^{*}(Y)\right)} \leq c_{\kappa} r_{\varepsilon, \lambda, \gamma}^{2} .
$$

Moreover,

$$
\begin{gathered}
\left\|\tilde{N}\left(\varepsilon, \lambda, \gamma, \tilde{\eta}, Y, \Phi, \Psi ; \tilde{v}_{2}\right)-\tilde{N}\left(\varepsilon, \lambda, \gamma, \tilde{\eta}, Y, \Phi, \Psi ; \tilde{v}_{1}\right)\right\|_{\mathcal{C}_{\nu}^{4, \alpha}\left(\bar{\Omega}^{*}(Y)\right)} \\
\leq \bar{c}_{\kappa} r_{\varepsilon, \lambda, \gamma}^{2}\left\|\tilde{v}_{2}-\tilde{v}_{1}\right\|_{\mathcal{C}_{\nu}^{4, \alpha}\left(\bar{\Omega}^{*}(Y)\right)}
\end{gathered}
$$

provided $\tilde{v}=v_{1}, v_{2} \in \mathcal{C}_{\nu}^{4, \alpha}\left(\bar{\Omega}^{*}(Y)\right), \tilde{\Phi}=\Phi_{1}, \Phi_{2} \in\left(\mathcal{C}^{4, \alpha}\left(S^{3}\right)\right)^{m}, \tilde{\Psi}=$ $\Psi_{1}, \Psi_{2} \in\left(\mathcal{C}^{2, \alpha}\left(S^{3}\right)\right)^{m}$ satisfy

$$
\|\tilde{v}\|_{\mathfrak{C}_{\nu}^{4, \alpha}\left(\bar{\Omega}^{*}(Y)\right)} \leq 2 c_{\kappa} r_{\varepsilon, \lambda, \gamma}^{2}, \quad\|\tilde{\Phi}\|_{\left(\mathfrak{C}^{4, \alpha}\left(S^{3}\right)\right)^{m}} \leq \kappa r_{\varepsilon, \lambda, \gamma}^{2}, \quad\|\tilde{\Psi}\|_{\left(\mathrm{e}^{2, \alpha}\left(S^{3}\right)\right)^{m}} \leq \kappa r_{\varepsilon, \lambda, \gamma}^{2},
$$

and $|\tilde{\eta}| \leq \kappa r_{\varepsilon, \lambda, \gamma}^{2},\|Y-X\| \leq \kappa r_{\varepsilon, \lambda, \gamma}$.

Proof. The proof of the first estimate follows from the asymptotic behavior of $H^{e}$ together with the assumption on the norm of boundary data $\tilde{\varphi}^{i}$ given by (52). Recall that a functions in $\mathcal{C}_{\nu}^{k, \alpha}\left(\bar{\Omega}^{*}(X)\right)$ are bounded by a constant times the distance to $X$ to the power $\nu$ and have their $\ell-t h$ partial derivatives that are bounded by a constant times the distance to $X$ to the power $\nu-\ell$, for $\ell=1, \cdots, k+\alpha$.

Indeed, let $c_{\kappa}$ be a constant depending only on $\kappa$ (provided $\varepsilon, \lambda$ and $\gamma$ are chosen small enough) it follows from the estimate of $H^{e}:=H_{\tilde{\varphi}^{j}, \tilde{\psi}^{j}}^{e}$ (Observe that (38) implies that the expansion of $H^{e}$ only involves powers of $r$ that are lower than or equal to -1 .), given by lemma 4.2 , then

$$
\left|H_{\tilde{\varphi}^{j}, \tilde{\psi}^{j}}^{e}\left(\left(x-y^{j}\right) / r_{\varepsilon, \lambda, \gamma}\right)\right| \leq c_{\kappa} r_{\varepsilon, \lambda, \gamma}^{3} r^{-1} \text {. }
$$

Recall that $\tilde{N}(\tilde{v})=\tilde{\mathcal{G}}_{\nu, Y} \circ \tilde{\mathcal{E}}_{r_{\varepsilon, \lambda, \gamma}, Y}(\tilde{S}(v))$, we will estimate $\tilde{N}(0)$ in different subregions of $\bar{\Omega}^{*}$. 
SINGULAR LIMITS FOR 4-DIMENSIONAL GENERAL STATIONARY Q-KURAMOTO-SIVASHINSKY EQUATION (Q-KSE) WITH EXPONENTIAL

- In $B_{r_{0} / 2}\left(y^{j}\right)$ for $1 \leq j \leq m$, we have $\chi_{r_{0}}\left(x-y^{j}\right)=1$ and $\Delta^{2} \tilde{\mathbf{u}}=0$, using Lemma 6.1 , so that

$$
\begin{aligned}
& |\tilde{S}(0)| \leq c_{\kappa} \varepsilon^{4} \prod_{j=1}^{m}\left[e^{\left(1+\tilde{\eta}^{j}\right) G_{y^{j}}(x)+H_{\tilde{\varphi}}^{e}, \tilde{\psi}^{j}}\left(\left(x-y^{j}\right) / r_{\varepsilon, \lambda, \gamma}\right)\right]+\gamma|\Delta \tilde{\mathbf{u}}|+\lambda|\nabla \tilde{\mathbf{u}}|^{q} \\
& \leq \quad c_{\kappa} \varepsilon^{4} \prod_{j=1}^{m}\left|x-y^{j}\right|^{-8\left(1+\tilde{\eta}^{j}\right)}+\gamma|\Delta(\tilde{\mathbf{u}})|+\lambda|\nabla(\tilde{\mathbf{u}})|^{q} \\
& \leq \quad c_{\kappa} \varepsilon^{4}\left|x-y^{j}\right|^{-8\left(1+\tilde{\eta}^{j}\right)} \prod_{\ell=1, \ell \neq j}^{m}\left|x-y^{\ell}\right|^{-8\left(1+\tilde{\eta}^{j}\right)} \\
& +c_{\kappa} \gamma \sum_{j=1}^{m}\left(1+\tilde{\eta}^{j}\right)\left|\Delta G\left(x, y^{j}\right)\right|+c_{\kappa} \gamma \sum_{j=1}^{m}\left|\left[\Delta, \chi_{r_{0}}\left(\cdot-y^{j}\right)\right]\left(H^{e}\left(\varphi^{j}, \psi^{j} ;\left(\cdot-y^{j}\right) / r_{\varepsilon, \lambda, \gamma}\right)\right)\right| \\
& +c_{\kappa} \lambda \sum_{j=1}^{m}\left(1+\tilde{\eta}^{j}\right)\left|\nabla G\left(x, y^{j}\right)\right|^{q}+c_{\kappa} \lambda \sum_{j=1}^{m}\left|\left[\nabla, \chi_{r_{0}}\left(\cdot-y^{j}\right)\right]\left(H^{e}\left(\varphi^{j}, \psi^{j} ;\left(\cdot-y^{j}\right) / r_{\varepsilon, \lambda, \gamma}\right)\right)\right|^{q} \\
& \leq c_{\kappa} \varepsilon^{4}\left|x-y^{j}\right|^{-8\left(1+\tilde{\eta}^{j}\right)} \prod_{\ell=1, \ell \neq j}^{m}\left|x-y^{\ell}\right|^{-8\left(1+\tilde{\eta}^{\ell}\right)}+c_{\kappa} \gamma\left(1+\tilde{\eta}^{j}\right)\left|x-y^{j}\right|^{-2} \\
& +\quad c_{\kappa} \gamma r_{\varepsilon, \lambda, \gamma}^{3}\left|x-y^{j}\right|^{-3}+c_{\kappa} \lambda\left(1+\tilde{\eta}^{j}\right)\left|x-y^{j}\right|^{-q}+c_{\kappa} \lambda r_{\varepsilon, \lambda, \gamma}^{3 q}\left|x-y^{j}\right|^{-2 q} .
\end{aligned}
$$

Here

$$
\left[\nabla, \chi_{r_{0}}\right] w=\nabla \chi_{r_{0}} \cdot w+\chi_{r_{0}} \cdot \nabla w
$$

and

$$
\left[\Delta, \chi_{r_{0}}\right] w=w \Delta \chi_{r_{0}}+\chi_{r_{0}} \Delta w+2 \nabla \chi_{r_{0}} \cdot \nabla w
$$

Hence, for $\nu \in(-1,0), q \in[1,4]$ and $\tilde{\eta}^{j}$ small enough, we get

$$
\begin{aligned}
\|\tilde{N}(0)\|_{\mathcal{C}_{\nu}^{4, \alpha}\left(\bigcup_{j=1}^{m} B\left(y^{j}, r_{0} / 2\right)\right)} & \leq \sup _{r_{\varepsilon, \lambda, \gamma} \leq r \leq r_{0} / 2} r^{4-\nu}|\tilde{N}(0)| \\
& \leq c_{\kappa} \varepsilon^{4} r_{\varepsilon, \lambda, \gamma}^{-4}+c_{\kappa} \gamma+c_{\kappa} \gamma r_{\varepsilon, \lambda, \gamma}^{3}+c_{\kappa} \lambda+c_{\kappa} \lambda r_{\varepsilon, \lambda, \gamma}^{4+q} \\
& \leq c_{\kappa} r_{\varepsilon, \lambda, \gamma}^{2} .
\end{aligned}
$$

- In $\Omega_{r_{0}, y^{j}}$ (recall that $\Omega_{r_{0}, y^{j}}=\Omega \backslash \cup_{j} B_{r_{0}}\left(y^{j}\right)$ ), we have $\chi_{r_{0}}\left(x-y^{j}\right)=0$ and $\Delta^{2} \tilde{\mathbf{u}}=0$, then

$$
\begin{aligned}
|\tilde{S}(0)| & \leq c_{\kappa} \varepsilon^{4}\left|x-y^{j}\right|^{-8\left(1+\tilde{\eta}^{j}\right)} \prod_{\ell=1, \ell \neq j}^{m}\left|x-y^{\ell}\right|^{-8\left(1+\tilde{\eta}^{j}\right)} \\
& +c_{\kappa} \gamma \sum_{j=1}^{m}\left(1+\tilde{\eta}^{j}\right)\left|\Delta G\left(x, y^{j}\right)\right|+c_{\kappa} \lambda \sum_{j=1}^{m}\left(1+\tilde{\eta}^{j}\right)\left|\nabla G\left(x, y^{j}\right)\right|^{q} \\
& \leq c_{\kappa} \varepsilon^{4}\left|x-y^{j}\right|^{-8\left(1+\tilde{\eta}^{j}\right)}+c_{\kappa} \gamma\left(1+\tilde{\eta}^{j}\right)\left|x-y^{j}\right|^{-2}+c_{\kappa} \lambda\left(1+\tilde{\eta}^{j}\right)\left|x-y^{j}\right|^{-q} .
\end{aligned}
$$


SINGULAR LIMITS FOR 4-DIMENSIONAL GENERAL STATIONARY

Q-KURAMOTO-SIVASHINSKY EQUATION (Q-KSE) WITH EXPONENTIAL

Thus

$$
\|\tilde{N}(0)\|_{\mathcal{C}_{\nu}^{4, \alpha}\left(\Omega_{r_{0}, \tilde{x}}\right)} \leq c_{\kappa} \sup _{r \geq r_{0}} r^{4-\nu}|\tilde{S}(0)| \leq c_{\kappa} \varepsilon^{4}+c_{\kappa} \gamma+c_{\kappa} \lambda .
$$

- In $B_{r_{0}}\left(y^{j}\right)-B_{r_{0} / 2}\left(y^{j}\right)$, for $j=1, \ldots, m$, taking into account that $\Delta^{2} G\left(x, y^{j}\right)=0$, we have

$$
\begin{aligned}
& |\tilde{S}(0)| \leq\left. c_{\kappa} \varepsilon^{4}\left|\prod_{j=1}^{m} e^{\left(1+\tilde{\eta}^{j}\right) G_{y^{j}}} e^{\chi_{r_{0}}\left(x-y^{j}\right) H_{\tilde{\varphi}^{j}, \tilde{\psi}^{j}}^{e}\left(\left(x-y^{j}\right) / r_{\varepsilon, \lambda, \gamma}\right)}-\Delta^{2} \tilde{\mathbf{u}}+\gamma \Delta \tilde{\mathbf{u}}+\lambda\right| \nabla \tilde{\mathbf{u}}\right|^{q} \mid \\
& \quad \leq c_{\kappa} \varepsilon^{4}\left|x-y^{j}\right|^{-8\left(1+\tilde{\eta}^{j}\right)} \prod_{\ell=1, \ell \neq 1}^{m}\left|x-y^{\ell}\right|^{-8\left(1+\tilde{\eta}^{\ell}\right)} \\
& +c_{\kappa} \sum_{j=1}^{m}\left(1+\tilde{\eta}^{j}\right)\left|\Delta^{2} G\left(x, y^{j}\right)\right|+c_{\kappa} \sum_{j=1}^{m}\left|\left[\Delta^{2}, \chi_{r_{0}}\left(x-y^{j}\right)\right]\left(H_{\tilde{\varphi}_{j}, \tilde{\psi}_{j}}^{e}\left(\left(x-y^{j}\right) / r_{\varepsilon, \lambda, \gamma}\right)\right)\right| \\
& +c_{\kappa} \gamma \sum_{j=1}^{m}\left(1+\tilde{\eta}^{j}\right)\left|\Delta G\left(x, y^{j}\right)\right|+c_{\kappa} \gamma \sum_{j=1}^{m}\left|\left[\Delta, \chi_{r_{0}}\left(\cdot-y^{j}\right)\right]\left(H^{e}\left(\varphi^{j}, \psi^{j} ;\left(\cdot-y^{j}\right) / r_{\varepsilon, \lambda, \gamma}\right)\right)\right| \\
& +c_{\kappa} \lambda \sum_{j=1}^{m}\left(1+\tilde{\eta}^{j}\right)\left|\nabla G\left(x, y^{j}\right)\right|^{q}+c_{\kappa} \lambda \sum_{j=1}^{m}\left|\left[\nabla, \chi_{r_{0}}\left(\cdot-y^{j}\right)\right]\left(H^{e}\left(\varphi^{j}, \psi^{j} ;\left(\cdot-y^{j}\right) / r_{\varepsilon, \lambda, \gamma}\right)\right)\right| \\
& \quad \leq \quad c_{\kappa} \varepsilon^{4}\left|x-y^{j}\right|^{-8\left(1+\tilde{\eta}^{j}\right)} \prod_{\ell=1, \ell \neq j}^{m}\left|x-y^{\ell}\right|^{-8\left(1+\tilde{\eta}^{\ell}\right)}+c_{\kappa} \gamma\left(1+\tilde{\eta}^{j}\right)\left|x-y^{j}\right|^{-2} \\
& \quad+c_{\kappa} \gamma r_{\varepsilon, \lambda, \gamma}^{3}\left|x-y^{j}\right|^{-3}+c_{\kappa} \lambda\left(1+\tilde{\eta}^{j}\right)\left|x-y^{j}\right|^{-q}+c_{\kappa} \lambda r_{\varepsilon, \lambda, \gamma}^{3 q}\left|x-y^{j}\right|^{-2 q} .
\end{aligned}
$$

Here

$\left[\Delta^{2}, \chi_{r_{0}}\right] w=2 \Delta \chi_{r_{0}} \Delta w+w \Delta^{2} \chi_{r_{0}}+4 \nabla \chi_{r_{0}} \cdot \nabla(\Delta w)+4 \nabla w \cdot \nabla\left(\Delta \chi_{r_{0}}\right)+4 \nabla^{2} \chi_{r_{0}} \cdot \nabla^{2} w$.

So, for $\left|x-y^{j}\right|=r$, we have $r_{0} / 2 \leq r \leq r_{0}$ then all quantity of type $\left|x-y^{j}\right|^{\ell}$, which appear to estimate $|\tilde{S}(0)|$ are bounded, then using(50) and Proposition 4.1 , we derive

$$
\begin{gathered}
\|\tilde{N}(0)\|_{\mathcal{C}_{\nu}^{4, \alpha}\left(B\left(y^{j}, r_{0}\right)-B\left(y^{j}, r_{0} / 2\right)\right)} \leq c_{\kappa} \sup _{r_{0} / 2 \leq r \leq r_{0}} r^{4-\nu}|\tilde{N}(0)| \\
\leq c_{\kappa} \varepsilon^{4}+c_{\kappa} \gamma+c_{\kappa} \gamma r_{\varepsilon, \lambda, \gamma}^{3}+c_{\kappa} \lambda+c_{\kappa} \lambda r_{\varepsilon, \lambda, \gamma}^{3 q} \leq c_{\kappa} r_{\varepsilon, \lambda, \gamma}^{2} .
\end{gathered}
$$

Finally in each subregions of $\bar{\Omega}^{*}$, we conclude that

$$
\|\tilde{N}(0)\|_{\mathcal{C}_{\nu}^{4, \alpha}\left(\Omega-\bigcup_{j=1}^{m} B\left(y^{j}, r_{\varepsilon, \lambda, \gamma}\right)\right)} \leq c_{\kappa} r_{\varepsilon, \lambda, \gamma}^{2} .
$$


SINGULAR LIMITS FOR 4-DIMENSIONAL GENERAL STATIONARY

Q-KURAMOTO-SIVASHINSKY EQUATION (Q-KSE) WITH EXPONENTIAL

To derive the second estimate, we use the fact that for $\left\|\tilde{v}_{i}\right\|_{C_{\nu}^{4, \alpha}\left(\bar{\Omega}^{*}\right)} \leq$ $c_{\kappa} r_{\varepsilon, \lambda, \gamma}^{2}$ for $i=1,2$ in different subregions of $\bar{\Omega}^{*}$, we derive the following estimates

$$
\begin{aligned}
\| \rho^{4}\left(e^{\tilde{\mathbf{u}}+v_{2}}\right. & \left.-e^{\tilde{\mathbf{u}}+v_{1}}\right) \|_{\mathcal{C}_{\nu-4}^{0, \alpha}\left(\bar{\Omega}_{r_{\varepsilon, \lambda, \gamma}}(Y)\right)} \\
& =\rho^{4} \sup _{r \in \bar{\Omega}_{r_{\varepsilon, \lambda, \gamma}}(Y)} r^{4-\nu}\left(e^{\tilde{\mathbf{u}}+\tilde{v}_{2}}-e^{\tilde{\mathbf{u}}+\tilde{v}_{1}}\right) \\
& \leq c_{\kappa} \varepsilon^{4}\left|\prod_{j=1}^{m} e^{\left(1+\tilde{\eta}^{j}\right) G_{y^{j}}} e^{\chi_{r_{0}}\left(x-y^{j}\right) H_{\tilde{\varphi}^{j}, \tilde{\psi}^{j}}^{e}\left(\left(x-y^{j}\right) / r_{\varepsilon, \lambda, \gamma}\right)}\left(e^{\tilde{v}_{1}}-e^{\tilde{v}_{2}}\right)\right| \\
& \leq c_{\kappa} \max \left(\varepsilon^{4} r_{\varepsilon, \lambda, \gamma}^{-4}, \varepsilon^{4}\right)\left\|\tilde{v}_{2}-\tilde{v}_{1}\right\|_{\mathcal{C}_{\nu}^{4, \alpha}\left(\bar{\Omega}^{*}(Y)\right)} \\
& \leq c_{\kappa} \varepsilon^{2}\left\|\tilde{v}_{2}-\tilde{v}_{1}\right\|_{\mathcal{C}_{\nu}^{4, \alpha}\left(\bar{\Omega}^{*}(Y)\right)}
\end{aligned}
$$

and the fact that for all $w \in \mathcal{C}_{\nu}^{4, \alpha}\left(\bar{\Omega}^{*}(Y)\right)$, there exist $c>0$ such that $|\Delta w| \leq$ $\operatorname{cr}^{\nu-2}\|w\|_{\mathcal{C}_{\nu}^{4, \alpha}\left(\bar{\Omega}^{*}(Y)\right)}$, then

$$
\begin{aligned}
\left\|\gamma\left(\Delta\left(\tilde{\mathbf{u}}+\tilde{v_{1}}\right)-\Delta\left(\tilde{\mathbf{u}}+\tilde{v_{2}}\right)\right)\right\|_{\mathcal{C}_{\nu}^{4, \alpha}\left(\bar{\Omega}^{*}(Y)\right)} & =c_{\kappa} \gamma \sup _{r \in \bar{\Omega}_{r_{\varepsilon, \lambda, \gamma}}(Y)} r^{4-\nu}\left|\Delta\left(\tilde{v}_{1}-\tilde{v_{2}}\right)\right| \\
& \leq c_{\kappa} \gamma \sup _{r \in \bar{\Omega}_{r_{\varepsilon, \lambda, \gamma}}(Y)} r^{2}\left\|\tilde{v}_{1}-\tilde{v}_{2}\right\|_{\mathcal{C}_{\nu}^{4, \alpha}\left(\bar{\Omega}^{*}\right)} \\
& \leq c_{\kappa} \gamma\left\|\tilde{v}_{1}-\tilde{v}_{2}\right\|_{\mathcal{C}_{\nu}^{4, \alpha}\left(\bar{\Omega}^{*}\right)}
\end{aligned}
$$

Again, making use of Lemma 3.2 and lemma 6.1 and the fact that for all $w \in \mathcal{C}_{\nu}^{4, \alpha}\left(\bar{\Omega}^{*}(Y)\right)$, there exist $c>0$ such that $|\nabla w| \leq c r^{\nu-1}\|w\|_{\mathcal{C}_{\nu}^{4, \alpha}\left(\bar{\Omega}^{*}(Y)\right)}$, we get

$$
\begin{aligned}
&\left\|\lambda\left|\nabla\left(\tilde{\mathbf{u}}+\tilde{v_{1}}\right)\right|^{q}-\lambda\left|\nabla\left(\tilde{\mathbf{u}}+\tilde{v_{2}}\right)\right|^{q}\right\|_{\mathcal{C}_{\nu}^{4, \alpha}\left(\bar{\Omega}^{*}(Y)\right)} \\
& \Rightarrow \sup _{r \in \bar{\Omega}_{r_{\varepsilon, \lambda, \gamma}}} r^{4-\nu}\left[\left|\nabla\left(\tilde{\mathbf{u}}+v_{1}\right)\right|^{q-1}+\left|\nabla\left(v_{1}-v_{2}\right)\right|^{q-1}\right]\left|\nabla\left(v_{2}-v_{1}\right)\right| \\
& \Rightarrow \sup _{r \in \bar{\Omega}_{r_{\varepsilon, \lambda, \gamma}}} r^{4-\nu}\left[|\nabla \tilde{\mathbf{u}}|^{q-1}+\left|\nabla v_{1}\right|^{q-1}+\left|\nabla v_{2}\right|^{q-1}\right]\left|\nabla\left(v_{2}-v_{1}\right)\right| \\
& \leq c_{\kappa} \lambda \sup _{r \in \bar{\Omega}_{r_{\varepsilon, \lambda, \gamma}}} r^{4-\nu} \mid \nabla\left(\sum_{j=1}^{m}\left(1+\tilde{\eta}^{j}\right) G\left(y^{j}, \cdot\right)\right. \\
&\left.\left.+\sum_{j=1}^{m} \chi_{r_{0}}\left(\cdot-y^{j}\right) H^{e}\left(\varphi^{j}, \psi^{j} ;\left(\cdot-y^{j}\right) / r_{\varepsilon, \lambda, \gamma}\right)\right)\right)\left.\right|^{q-1}\left|\nabla\left(v_{2}-v_{1}\right)\right|
\end{aligned}
$$




$$
\begin{aligned}
& +c_{\kappa} \lambda \sup _{r \in \bar{\Omega}_{r_{\varepsilon, \lambda, \gamma}}} r^{4-\nu} \sum_{i=1}^{2}\left\|\tilde{v}_{i}\right\|_{C_{\nu}^{4}, \alpha\left(\bar{\Omega}^{*}\right)}^{q-1}\left|\nabla\left(v_{2}-v_{1}\right)\right| \\
& \leq c_{\kappa} \lambda\left(1+\tilde{\eta}^{j}\right) c_{\kappa} \sup _{r \in \bar{\Omega}_{r_{\varepsilon, \lambda, \gamma}}} r^{3} \sum_{j=1}^{m}\left|\nabla G\left(y^{j}, \cdot\right)\right|^{q-1}\left\|\tilde{v}_{2}-\tilde{v}_{1}\right\|_{C_{\nu}^{4, \alpha}\left(\bar{\Omega}^{*}\right)} \\
& +\lambda \sup _{r \in \bar{\Omega}_{r_{\varepsilon, \lambda, \gamma}}} r^{3} \sum_{j=1}^{m}\left|\left[\nabla, \chi_{r_{0}}\left(x-y^{j}\right)\right]\left(H_{\tilde{\varphi}_{j}, \tilde{\psi}_{j}}^{e}\left(\left(x-y^{j}\right) / r_{\varepsilon, \lambda, \gamma}\right)\right)\right|^{q-1}\left\|\tilde{v}_{2}-\tilde{v}_{1}\right\|_{C_{\nu}^{4}, \alpha\left(\bar{\Omega}^{*}\right)} \\
& +c_{\kappa} \lambda \sup _{r \in \bar{\Omega}_{r_{\varepsilon, \lambda, \gamma}}} r^{3} \sum_{i=1}^{2}\left\|\tilde{v}_{i}\right\|_{C_{\nu}^{4, \alpha}\left(\bar{\Omega}^{*}\right)}^{q-1}\left\|\tilde{v}_{2}-\tilde{v}_{1}\right\|_{C_{\nu}^{4, \alpha}\left(\bar{\Omega}^{*}\right)} \\
& \leq c_{\kappa} \lambda\left(1+\tilde{\eta}^{j}\right) c_{\kappa} \sup _{r \in \bar{\Omega}_{r_{\varepsilon, \lambda, \gamma}}} r^{3} r^{1-q}\left\|\tilde{v}_{2}-\tilde{v}_{1}\right\|_{C_{\nu}^{4, \alpha}\left(\bar{\Omega}^{*}\right)} \\
& +\quad c_{\kappa} \lambda \sup _{r \in \bar{\Omega}_{r, \lambda, \gamma}} r^{3} r_{\varepsilon, \lambda, \gamma}^{3(q-1)} r^{-2(q-1)}\left\|\tilde{v}_{2}-\tilde{v}_{1}\right\|_{C_{\nu}^{4, \alpha}\left(\bar{\Omega}^{*}\right)} \\
& +2 c_{\kappa} \lambda r_{\varepsilon, \lambda, \gamma}^{2(q-1)} \sup _{r \in \bar{\Omega}_{r_{\varepsilon, \lambda, \gamma}}} r^{3}\left\|\tilde{v}_{2}-\tilde{v}_{1}\right\|_{C_{\nu}^{4, \alpha}\left(\bar{\Omega}^{*}\right)}
\end{aligned}
$$

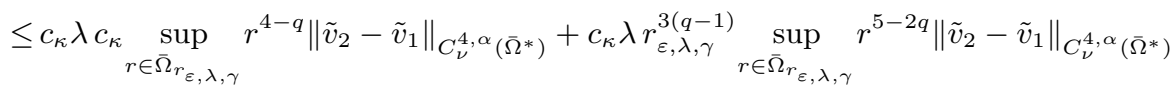

$$
\begin{aligned}
& +2 c_{\kappa} \lambda r_{\varepsilon, \lambda, \gamma}^{2(q-1)} \sup _{r \in \bar{\Omega}_{r_{\varepsilon, \lambda, \gamma}}} r^{3}\left\|\tilde{v}_{2}-\tilde{v}_{1}\right\|_{C_{\nu}^{4, \alpha}\left(\bar{\Omega}^{*}\right)} \\
& \leq c_{\kappa} \lambda\left(1+r_{\varepsilon, \lambda, \gamma}^{3(q-1)}+\max \left(r_{\varepsilon, \lambda, \gamma}^{3(q-1)}, r_{\varepsilon, \lambda, \gamma}^{q+2}\right)+r_{\varepsilon, \lambda, \gamma}^{2(q-1)}\right)\left\|\tilde{v}_{2}-\tilde{v}_{1}\right\|_{C_{\nu}^{4, \alpha}\left(\bar{\Omega}^{*}\right)} \\
& \leq c_{\kappa}\left\|\tilde{v}_{2}-\tilde{v}_{1}\right\|_{C_{\nu}^{4, \alpha}\left(\bar{\Omega}^{*}\right)} \text {. }
\end{aligned}
$$

Using(50) and Proposition 4.1, we conclude that

$$
\left\|\tilde{N}\left(\tilde{v}_{1}\right)-\tilde{N}\left(\tilde{v}_{2}\right)\right\|_{\mathcal{C}_{\nu}^{4, \alpha}\left(\Omega_{r_{\varepsilon, \lambda, \gamma}, y^{j}}\right)} \leq c_{\kappa} r_{\varepsilon, \lambda, \gamma}^{2}\left\|\tilde{v}_{1}-\tilde{v}_{2}\right\|_{\mathcal{C}_{\nu}^{4, \alpha}\left(\bar{\Omega}^{*}\right)} .
$$

Reducing $\varepsilon_{\kappa}, \lambda_{\kappa}$ and $\gamma_{\kappa}$ if necessary, we can assume that

$$
\bar{c}_{\kappa} r_{\varepsilon, \lambda, \gamma}^{2} \leq \frac{1}{2}
$$

for all $\varepsilon \in\left(0, \varepsilon_{\kappa}\right), \lambda \in\left(0, \lambda_{\kappa}\right)$ and $\gamma \in\left(0, \gamma_{\kappa}\right)$. Then, (54) and (55) are enough to show that

$$
\tilde{v} \longmapsto \tilde{N}(\varepsilon, \lambda, \gamma, \tilde{\eta}, Y, \Phi, \Psi \tilde{v})
$$

is a contraction from

$$
\left\{\tilde{v} \in \mathcal{C}_{\nu}^{4, \alpha}\left(\bar{\Omega}^{*}(Y)\right) \quad: \quad\|\tilde{v}\|_{\mathcal{C}_{\nu}^{4, \alpha}\left(\bar{\Omega}^{*}(Y)\right)} \leq 2 c_{\kappa} r_{\varepsilon, \lambda, \gamma}^{2}\right\}
$$

into itself and hence has a unique fixed point $\tilde{v}(\varepsilon, \tilde{\eta}, Y, \Phi, \Psi ; \cdot)$ in this set. This fixed point is a solution of (49).

We summarize this in the : 
SINGULAR LIMITS FOR 4-DIMENSIONAL GENERAL STATIONARY

Proposition 6.1. Given $\kappa>0$, there exists $\varepsilon_{\kappa}>0, \lambda_{\kappa}>0, \gamma_{\kappa}>0$, and $c_{\kappa}>$ 0 (only depending on $\kappa$ ) such that for all $\varepsilon \in\left(0, \varepsilon_{\kappa}\right), \lambda \in\left(0, \lambda_{\kappa}\right), \gamma \in\left(0, \gamma_{\kappa}\right)$ (satisfying condition $\left(A_{\varepsilon, \lambda, \gamma}\right)$, when $q=4$ ) and for all set of parameters $\tilde{\eta}$, points $Y$ satisfying

$$
|\tilde{\eta}| \leq \kappa r_{\varepsilon, \lambda, \gamma}^{2}, \quad \text { and } \quad\|Y-X\| \leq \kappa r_{\varepsilon, \lambda, \gamma}
$$

and boundary functions $\Phi$ and $\Psi$ satisfying (38) and

$$
\|\Phi\|_{\left(\mathcal{C}^{4, \alpha}\left(S^{3}\right)\right)^{m}} \leq \kappa r_{\varepsilon, \lambda, \gamma}^{2}, \quad \text { and } \quad\|\Psi\|_{\left(\mathcal{C}^{2, \alpha}\left(S^{3}\right)\right)^{m}} \leq \kappa r_{\varepsilon, \lambda, \gamma}^{2} .
$$

the function

$$
\begin{aligned}
\tilde{u}(\varepsilon, \lambda, \gamma, \tilde{\eta}, Y, \Phi, \Psi ; \cdot): & =\sum_{j=1}^{m}\left(1+\tilde{\eta}^{j}\right) G_{y^{j}}+\sum_{j=1}^{m} \chi_{r_{0}}\left(\cdot-y^{j}\right) H^{e}\left(\varphi^{j}, \psi^{j} ;\left(\cdot-y^{j}\right) / r_{\varepsilon, \lambda, \gamma}\right) \\
& +\tilde{v}(\varepsilon, \lambda, \gamma, \tilde{\eta}, Y, \Phi, \Psi ; \cdot),
\end{aligned}
$$

solves (48) in $\bar{\Omega}_{r_{\varepsilon, \lambda, \gamma}}(Y)$. In addition

$$
\|\tilde{v}(\varepsilon, \lambda, \gamma, \tilde{\eta}, Y, \Phi, \Psi ; \cdot)\|_{\mathcal{C}_{\nu}^{4, \alpha}\left(\bar{\Omega}^{*}\right)} \leq 2 c_{\kappa} r_{\varepsilon, \lambda, \gamma}^{2} .
$$

Observe that the function $\tilde{v}_{\varepsilon, \lambda, \gamma, \tilde{\eta}, Y, \Phi, \Psi}$ being obtained as a fixed point for contraction mapping, it depends continuously on the parameters $\tilde{\eta}$ and the points $Y$.

\section{The nonlinear Cauchy-data matching}

Keeping the notations of the previous sections, we gather the results of the Proposition 5.1 and Proposition 6.1. From now let $\kappa>1$ is fixed large enough (we will shortly see how) and assume that $\varepsilon \in\left(0, \varepsilon_{\kappa}\right), \lambda \in\left(0, \lambda_{\kappa}\right)$ and $\gamma \in$ $\left(0, \gamma_{\kappa}\right)$ (satisfying condition $\left(A_{\varepsilon, \lambda, \gamma}\right)$, when $\left.q=4\right)$.

Assume that $X=\left(x^{1}, \ldots, x^{m}\right) \in \Omega^{m}$ is a nondegenerate critical point of the function $W$ defined in the introduction. For all $j=1, \ldots, m$, we define $\tau_{*}^{j}>0$ by

$$
-4 \log \tau_{*}^{j}=R\left(x^{j}, x^{j}\right)+\sum_{\ell \neq j} G\left(x^{\ell}, x^{j}\right) .
$$

We assume that we are given :

(i) points $Y:=\left(y^{1}, \ldots, y^{m}\right) \in \Omega^{m}$ close to $X:=\left(x^{1}, \ldots, x^{m}\right)$ satisfying (53). 
SINGULAR LIMITS FOR 4-DIMENSIONAL GENERAL STATIONARY

Q-KURAMOTO-SIVASHINSKY EQUATION (Q-KSE) WITH EXPONENTIAL

NONLINEARITY

(ii) parameters $\tilde{\eta}:=\left(\tilde{\eta}^{1}, \ldots, \tilde{\eta}^{m}\right) \in \mathbb{R}^{m}$ satisfying (53).

(iii) parameters $T:=\left(\tau^{1}, \ldots, \tau^{m}\right) \in(0, \infty)^{m}$ satisfying (42) (where, for each $j=1, \ldots, m, \tau_{*}$ is replaced by $\left.\tau_{*}^{j}\right)$.

We set

$$
R_{\varepsilon, \lambda, \gamma}^{j}:=\tau^{j} / r_{\varepsilon, \lambda, \gamma}
$$

First, we consider some set of boundary data

$\Phi:=\left(\varphi^{1}, \ldots, \varphi^{m}\right) \in\left(\mathcal{C}^{4, \alpha}\left(S^{3}\right)\right)^{m} \quad$ and $\quad \Psi:=\left(\psi^{1}, \ldots, \psi^{m}\right) \in\left(\mathcal{C}^{2, \alpha}\left(S^{3}\right)\right)^{m}$ satisfying (36) and (42).

Thanks to the result of Proposition 5.1, we can find $u_{\text {int }}$ a solution of

$$
\Delta^{2} u-\gamma \Delta u-\lambda|\nabla u|^{q}-\rho^{4} e^{u}=0
$$

in each $B_{r_{\varepsilon, \lambda, \gamma}}\left(y^{j}\right)$, which can be decomposed as

$$
\begin{aligned}
u_{\text {int }}(\varepsilon, \lambda, \gamma, T, Y, \Phi, \Psi ; x):= & u_{\varepsilon, \tau^{j}}\left(x-y^{j}\right)+h\left(R_{\varepsilon, \lambda, \gamma}^{j}\left(x-y^{j}\right) / r_{\varepsilon, \lambda, \gamma}\right) \\
& +H^{i}\left(\varphi^{j}, \psi^{j} ;\left(x-y^{j}\right) / r_{\varepsilon, \lambda, \gamma}\right) \\
& +v\left(\varepsilon, \lambda, \gamma, \tau^{j}, \varphi^{j}, \psi^{j} ; R_{\varepsilon, \lambda, \gamma}^{j}\left(x-y^{j}\right) / r_{\varepsilon, \lambda, \gamma}\right)
\end{aligned}
$$

in $B_{r_{\varepsilon, \lambda, \gamma}}\left(y^{j}\right)$ where, for $q \in[1,4)$ and $\delta \in(0, \min (1,4-q))$,

$$
\|h\|_{\mathcal{C}_{r a d, \delta}^{4, \alpha}\left(\mathbb{R}^{4}\right)} \leq 2 c_{\kappa} \varepsilon^{\delta} r_{\varepsilon, \lambda, \gamma}^{2}
$$

and for $q=4, h:=\hbar$ and $\delta \in(0,1)$,

$$
\|\hbar\|_{\mathcal{E}_{r a d, \delta}^{4, \alpha}\left(\mathbb{R}^{4}\right)} \leq 2 c_{\kappa} r_{\varepsilon, \lambda, \gamma}^{2} .
$$

Similarly, given some boundary data

$\tilde{\Phi}:=\left(\tilde{\varphi}^{1}, \ldots, \tilde{\varphi}^{m}\right) \in\left(\mathcal{C}^{4, \alpha}\left(S^{3}\right)\right)^{m} \quad$ and $\quad \tilde{\Psi}:=\left(\tilde{\psi}^{1}, \ldots, \tilde{\psi}^{m}\right) \in\left(\mathcal{C}^{2, \alpha}\left(S^{3}\right)\right)^{m}$ satisfying (38) and (52), we use the result of Proposition 6.1, to find $u_{e x t}$ a solution of

$$
\Delta^{2} u-\lambda \Delta u-\gamma|\nabla u|^{q}-\rho^{4} e^{u}=0
$$


SINGULAR LIMITS FOR 4-DIMENSIONAL GENERAL STATIONARY

in $\bar{\Omega}_{r_{\varepsilon, \lambda, \gamma}}(Y)$, which can be decomposed as

$$
\begin{aligned}
u_{e x t}(\varepsilon, \lambda, \gamma, \tilde{\eta}, \tilde{\Phi}, \tilde{\Psi} ; x) & :=\sum_{j=1}^{m}\left(1+\tilde{\eta}^{j}\right) G\left(y^{j}, x\right) \\
& +\sum_{j=1}^{m} \chi_{r_{0}}\left(x-y^{j}\right) H^{e}\left(\tilde{\varphi}^{j}, \tilde{\psi}^{j} ;\left(x-y^{j}\right) / r_{\varepsilon, \lambda, \gamma}\right) \\
& +\tilde{v}(\varepsilon, \lambda, \gamma, \tilde{\eta}, Y, \tilde{\Phi}, \tilde{\Psi} ; x) .
\end{aligned}
$$

It remains to determine the parameters and the boundary functions in such a way that the function which is equal to $u_{\text {int }}$ in $\cup_{j} B_{r_{\varepsilon, \lambda, \gamma}}\left(y^{j}\right)$ and which is equal to $u_{e x t}$ in $\bar{\Omega}_{r_{\varepsilon, \lambda, \gamma}}(Y)$ is a smooth function. This amounts to find the boundary data and the parameters so that, for each $j=1, \ldots, m$

$u_{\text {int }}=u_{\text {ext }}, \quad \partial_{r} u_{\text {int }}=\partial_{r} u_{\text {ext }}, \quad \Delta u_{i n t}=\Delta u_{\text {ext }}, \quad \partial_{r} \Delta u_{i n t}=\partial_{r} \Delta u_{\text {ext }}$,

on $\partial B_{r_{\varepsilon, \lambda, \gamma}}\left(y^{j}\right)$. Assuming we have already done so, this provides for each $\varepsilon, \lambda$ and $\gamma$ are small enough, a function $w_{\varepsilon, \lambda, \gamma} \in \mathcal{C}^{4, \alpha}(\bar{\Omega})$ (which is obtained by patching together the function $u_{\text {int }}$ and the function $u_{e x t}$ ) solution of $\Delta^{2} u-\gamma \Delta u-\lambda|\nabla u|^{q}-\rho^{4} e^{u}=0$ and elliptic regularity theory implies that this solution is in fact smooth. This will complete the proof of our result since, as $\varepsilon, \lambda$ and $\gamma$ tend to 0 , the sequence of solutions we have obtained satisfies the required properties, namely, away from the points $x^{j}$ the sequence $w_{\varepsilon, \lambda, \gamma}$ converges to $\sum_{j} G\left(x^{j}, \cdot\right)$. This completes the proof of Theorem 1.2 and Theorem 1.3. We leave the details to the reader (For more details see [1]).

\section{Acknowledgments}

The authors would like to thank Deanship of Scientific Research at Majmaah University, Saudi Arabia for the financial grant received in conducting this research.

\section{References}

[1] S. Baraket, M. Dammak, T. Ouni and F. Pacard, Singular limits for a 4-dimensional semilinear elliptic problem with exponential nonlinearity, Ann. I. H. Poincaré - AN 24 (2007), 875-895.

[2] S. Baraket, I. Ben Omrane and T. Ouni, Singular limits for 2-dimensional elliptic problem involving exponential with nonlinear gradient term. Nonlinear Differ. Equ. Appl. 18, 59-78 (2011).

[3] S. Baraket and T. Ouni, Singular limits for a 2-dimensional semilinear elliptic problem involving exponential nonlinearites with sub-quadratic 
SINGULAR LIMITS FOR 4-DIMENSIONAL GENERAL STATIONARY

Q-KURAMOTO-SIVASHINSKY EQUATION (Q-KSE) WITH EXPONENTIAL

NONLINEARITY

convection nonlinear gradient terms, Glasgow Math. J. Trust (2013): 121.

[4] S. Baraket, Moufida Khtaifi and T. Ouni, Singular limits solution for 4-dimensional stationary Kuramoto-Sivashinsky equation with exponential nonlinearity, Electronic Journal of Differential Equations, Vol. 2015 (2015), No. 187, pp. 1-26.

[5] H. Bellout, S. Benachour, and E. S. Titi, Finite-time singularity versus global regularity for hyper-viscous Hamilton-Jacobi-like equations. Nonlinearity, 16(6) (2003), 1967-1989.

[6] S.Y.A. Chang, On a fourth order differential operator - the Paneitz operator - in conformal geometry, preprint, to appear in the preceedings conference for the $70^{t h}$ birthday of A.P. Calderon.

[7] S.Y.A. Chang and P. Yang, On a fourth order curvature invariant, Comtemporary Mathematics ,237, Spectral Problems in Geometry and Arithmetic, Ed. T.Branson, AMS, 1999, 9-28.

[8] A. Cheskidov, C. Foias, On non-homogeneous KuramotoSivashinsky equation, Physica D: Nonlinear Phenomena Volume 154, Issues 1-2, 1 June 2001, Pages 1-14

[9] Dimitri Mugnai, A note on an exponential semilinear equation of the fourth order, Differential Integral Equations Volume 17, Number 1-2 (2004), 45-52.

[10] A. Malchiodi and Z. Djadli, Existence of conformal metrics with constant Q-curvature, Preprint math.AP/0410141.

[11] P. Esposito, M. Musso and A. Pistoia, Concentrating solutions for a planar problem involving nonlinearities with large exponent, J. Diff. Eqns, 227 (2006), 29-68.

[12] Ju.P. Krasovski $\breve{i}$, Isolation of singularities of the Greens function, (Russian), Izv. Akad. Nauk SSSR Ser. Mat., 31, 977-1010 (1967), English translation in: Math. USSR, Izv., 1, 935-966 (1967).

[13] Y. Kuramoto, T. Tsuzuki Y. Kuramoto, T. Tsuzuki, Persistent propagation of concentration waves in dissipative media far from thermal equilibrium, Prog. Theor. Phys. 55 (1976) 356-369.

[14] R. Mazzeo, Elliptic theory of edge operators I. Comm. in PDE No 16, 10 (1991) 1616-1664. 
SINGULAR LIMITS FOR 4-DIMENSIONAL GENERAL STATIONARY

Q-KURAMOTO-SIVASHINSKY EQUATION (Q-KSE) WITH EXPONENTIAL

NONLINEARITY

[15] R. Melrose, The Atiyah-Patodi-Singer index theorem, Research notes in Math 4 (1993).

[16] F. Pacard and T. Rivière, Linear and nonlinear aspects of vortices : the Ginzburg Landau model Progress in Nonlinear Differential Equations, 39, Birkäuser (2000).

[17] M. Raible, S. Linz, and P. Hanggi,Amorphous thin film growth: modeling and pattern formation, Advances in Solid State Physics, 41:391-403,2001.

[18] M. Raible, S. Mayr, S. Linz, M. Moske, P. Hanggi, and K. Samwer , Amorphous thin film growth: theory compared with experiment, Europhysics Letters, 50:6167,2000.

[19] X. Ren and J. Wei, On a two-dimensional elliptic problem with large exponent in nonlinearity, Trans. Amer. Math. Soc. 343 (1994), 749-763.

[20] Ruf, B, Sani, F, Sharp Adams-type inequalities in $R^{N}$, Trans. Am. Math. Soc. 365, 645-670 (2013)

[21] Ruichang Pei and Jihui Zhang, Biharmonic equations with improved subcritical polynomial growth and subcritical exponential growth, Boundary Value Problems 2014, 2014:162.

[22] P.Souplet, Gradient blow up for multidimensional nonlinear parabolic equations with general boundary condition. Differential Integral Equations 15 (2002) 237-256. Monge-Ampère equation, Springer Verlag, York, 1983.

[23] Y. Rébai, Weak solutions of nonlinear elliptic with prescribed singular set, J. Differ. Equations, 127 (1996), no. 2, 439-453.

[24] G. Sivashinsky G. Sivashinsky, Nonlinear analysis of hydrodynamic instability in laminar flame. I. Derivation of basic equations, Acta Astron. 4 (1977) 1117-1206.

[25] G. Sivashinsky G. Sivashinsky, Weak turbulence in periodic flows, Physica D 17 (1985) 243255.

[26] J. Wei, Asymptotic behavior of a nonlinear fourth order eigenvalue problem, Comm. Partial Differential Equations, 21(9-10) (1996), 1451-1467.

[27] H.C. Wente, Counter example to a conjecture of H. Hopf, Pacific J.Math.121 (1986), 193-243. 
SINGULAR LIMITS FOR 4-DIMENSIONAL GENERAL STATIONARY

Q-KURAMOTO-SIVASHINSKY EQUATION (Q-KSE) WITH EXPONENTIAL

NONLINEARITY

Taieb Ouni

Department of Mathematics, College of Sciences and Human

Studies at Howtat Sudair, Majmaah University, 11982, Saudi Arabia.

E-mail address: t.ouni@mu.edu.sa, taieb.ouni@fst.rnu.tn

Sami Baraket

Département de Mathématiques, Faculté des Sciences de Tunis.

Campus Universitaire, 2092 Tunis, Tunisia

E-mail address: smbaraket@yahoo.fr

Moufida Khtaifi

Département de Mathématiques, Faculté des Sciences de Tunis.

Campus Universitaire, 2092 Tunis, Tunisia

E-mail address: moufida180888@gmail.com 